for

\title{
Synthesis of 3-substituted 2-fluoro- and 2,2-difluoroaziridines
}

\author{
Eva Van Hende, Guido Verniest, ${ }^{\S}$ Riccardo Surmont and Norbert De Kimpe ${ }^{* *}$ \\ Department of Organic Chemistry, Faculty of Bioscience Engineering, \\ Ghent University, Coupure Links 653, 9000 Ghent, Belgium
}

TABLE OF CONTENTS:

I. General methods

II. Experimental procedures and spectroscopic data $\left({ }^{1} \mathrm{H}\right.$ NMR, ${ }^{13} \mathrm{C}$ NMR,

${ }^{19} \mathrm{~F}$ NMR, IR, MS, HRMS and elemental analysis) of all new compounds

2g, 3a-h, 4a-e, 5a-e, 6a-c, 6e, 9a-e, 11a-d, 12a-d, 15a-b and 16a-b

1. Synthesis of $\alpha$-chloro- $\alpha(, \alpha)$-(di)fluoroketones $\mathbf{2}$ and $\mathbf{8}$

2. Synthesis of $\alpha(, \alpha)$-(di)chloro- and $\alpha(, \alpha)$-(di)bromo- $\alpha(, \alpha)$-(di)fluoroimines 3a-h,

9a-e

2.1. By imine formation of the corresponding $\alpha$-chloro- $\alpha(, \alpha)$-(di)fluoroketones 3d, e, 9a-

d

2.2. By chlorination or bromination of the corresponding $\alpha(, \alpha)$-(di)fluoroimines

3a-c, 3f-h, 9e.

3. Synthesis of $\beta$-chloro and $\beta$-bromo- $\beta(, \beta)$-(di)fluoroamines 11a-d and 4a-e

4. Synthesis of monofluoroaziridines 5a-e and 6a-e

5. Synthesis of 2,2-difluoroaziridines 12a-d

6. Synthesis of amides $\mathbf{1 5}$ and $\mathbf{1 6}$

III. Copies of ${ }^{1} \mathrm{H}$ NMR and ${ }^{13} \mathrm{C}$ NMR spectra of $\mathbf{2 g}$, 3a-h, 4a-e, 5a-e, 6a-c, 6e, 8a-b, 9a-e, 11a-d, 12a-d, 15a-b and 16a-b

\footnotetext{
$\S$ Postdoctoral Fellow of the Research Foundation - Flanders (FWO - Vlaanderen)

** Corresponding author. Tel.: +32 (0)9 26459 51; fax: +32 (0)9 26462 43; e-mail: norbert.dekimpe@UGent.be
} 


\section{General methods}

${ }^{1} \mathrm{H}$ NMR (300 MHz), ${ }^{13} \mathrm{C}$ NMR (75 MHz) and ${ }^{19} \mathrm{~F}$ NMR (282 MHz) spectra were run with a Jeol JNM-EX 270 NMR spectrometer or with a Jeol Eclipse FT 300 NMR spectrometer. The compounds were dissolved in $\mathrm{CDCl}_{3}$ with tetramethylsilane (TMS, $\delta=0 \mathrm{ppm}$ ) as reference.

${ }^{19} \mathrm{~F}$ NMR spectra were recorded using $\mathrm{CDCl}_{3}$ as lock solvent. IR spectra were obtained from a Perkin Elmer Spectrum One infrared spectrometer. Mass spectra were recorded on a Varian MAT 112 spectrometer $(70 \mathrm{eV})$, using either GC-MS coupling or the direct inlet system. Some volatile compounds were recorded on a HP 6890 GC coupled with a HP 5973 MSD (Mass selective detector). LC-MS was performed with an Agilent 1100 Series VS (ES, 4000V) mass spectrometer. HRMS analyses were performed using a Finnigan MAT 95 XP-API-GC Trap tandem mass spectrometer system. Melting points of crystalline compounds were measured with a Büchi 540 apparatus. Elemental analysis of several new compounds was performed on a Perkin Elmer 2400 Elemental Analyser.

\section{Synthetic procedures and spectral data}

\section{Synthesis of $\alpha$-chloro- $\alpha(, \alpha)$-(di)fluoroketones 2 and 8}

2-Chloro-2-fluoro-1-(4-methoxyphenyl)ethanone $2 \mathrm{~d}$. The synthesis of $\alpha$-chloro- $\alpha$ fluoroketone $\mathbf{2 d}$ is given as a representative example for the synthesis of both $\alpha$-chloro- $\alpha(, \alpha)$ (di)fluoroketones $\mathbf{2 d}$ and $\mathbf{8}$ which are used as starting material for further imine formation reactions. Spectral data of known ketones $2 \mathbf{e}^{\dagger \dagger}$ and $\mathbf{8 a - c} \mathbf{c}^{\dagger}$ were in accordance with literature data.

4-Methoxyphenylmagnesium bromide was prepared from $24.32 \mathrm{~g}$ of 4-bromoanisole (130 mmol, 1.3 equiv) and $3.20 \mathrm{~g}$ of magnesium (130 mmol, 1.3 equiv) in $20 \mathrm{~mL}$ of diethyl ether (dry). This Grignard reagent was added via a syringe to a solution of $14.04 \mathrm{~g}$ of ethyl chlorofluoroacetate $(100 \mathrm{mmol})$ in diethyl ether $(30 \mathrm{~mL})$ under nitrogen at $-78{ }^{\circ} \mathrm{C}$. After stirring for $2 \mathrm{~h}$ at $-78^{\circ} \mathrm{C}$, the reaction mixture was hydrolyzed with aqueous $\mathrm{HCl}(6 \mathrm{M}, 10 \mathrm{~mL})$ below $0{ }^{\circ} \mathrm{C}$ and stirred for $1 \mathrm{~h}$ at room temperature. The resulting mixture was extracted with

\footnotetext{
† De Kimpe, N.; Verhé, R.; De Buck, L.; Schamp, N. Tetrahedron Lett. 1980, 21, 2257.

(a) Qiu, Z.-M.; Burton, D.J. J. Org. Chem. 1995, 60, 5570. (b) Kuroboshi, M.; Ishihara, T. Bull. Chem. Soc. Jpn., 1990, 63, 428.
} 
diethyl ether ( $3 \times 100 \mathrm{~mL})$ and the combined organic phases were washed with a saturated $\mathrm{NaHCO}_{3}$ aqueous solution $(2 \times 100 \mathrm{~mL})$ and brine $(2 \times 100 \mathrm{~mL})$, and subsequently dried over $\mathrm{MgSO}_{4}$. After evaporation of the solvent in vacuo, the crude ketone $2 \mathbf{d}$ was distilled. Bp 145 ${ }^{\circ} \mathrm{C}(12 \mathrm{mmHg})$, yield 20\%. Yellowish oil. ${ }^{1} \mathbf{H}$ NMR $\left(300 \mathrm{MHz}, \mathrm{CDCl}_{3}\right): \delta 3.89\left(3 \mathrm{H}, \mathrm{s}, \mathrm{CH}_{3} \mathrm{O}\right)$; $6.80(1 \mathrm{H}, \mathrm{d}, \mathrm{J}=50.5 \mathrm{~Hz}, \mathrm{CHClF}) ; 6.96\left(2 \mathrm{H}, \mathrm{d}, \mathrm{J}=9.1 \mathrm{~Hz}, 2 \mathrm{xCH}_{\mathrm{ar}}\right) ; 8.05(2 \mathrm{H}, \mathrm{d}, \mathrm{J}=9.1 \mathrm{~Hz}$, $\left.2 \mathrm{xCH}_{\mathrm{ar}}\right) .{ }^{19} \mathbf{F}$ NMR $\left(282 \mathrm{MHz}, \mathrm{CDCl}_{3}\right): \delta-145.5(1 \mathrm{~F}, \mathrm{~d}, \mathrm{~J}=50.5 \mathrm{~Hz}) .{ }^{13} \mathbf{C} \mathbf{N M R}(75 \mathrm{MHz}$, $\mathrm{CDCl}_{3}$, int. ref. $\left.=77.26 \mathrm{ppm}\right): \delta 55.8\left(\mathrm{CH}_{3} \mathrm{O}\right) ; 95.5(\mathrm{~d}, \mathrm{~J}=257.3 \mathrm{~Hz}, \mathrm{CFCl}) ; 114.4\left(2 \mathrm{xCH}_{\mathrm{ar}}\right)$; $124.1\left(\mathrm{C}_{\text {ar,quat }}\right) ; 132.3\left(2 \mathrm{xCH}_{\mathrm{ar}}\right) ; 165.0\left(\mathrm{C}_{\mathrm{ar}, \text { quat }}\right) ; 186.2(\mathrm{~d}, \mathrm{~J}=23.1 \mathrm{~Hz}, \mathrm{C}=\mathrm{O}) . \mathbf{I R}\left(\mathrm{NaCl}, \mathrm{cm}^{-1}\right)$ :

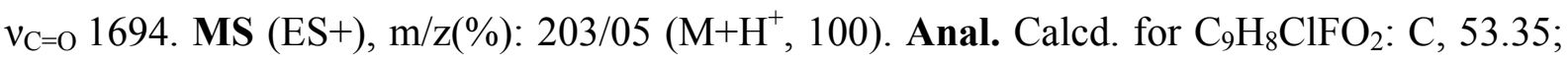
H, 3.98; N, 0.00. Found: C, 52.98; H, 4.07; N, 0.26. Copies of ${ }^{1} \mathrm{H}$ NMR and ${ }^{13} \mathrm{C}$ NMR spectra are included in chapter III of the supporting information file.

Copies of ${ }^{1} \mathrm{H}$ NMR and ${ }^{13} \mathrm{C}$ NMR spectra of ketones $2 \mathbf{d}, \mathbf{8 a}-\mathbf{b}$ are included in chapter III of the supporting information file.

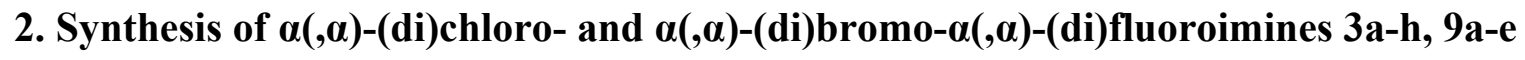
e, 9 a-d

2.1. By imine formation of the corresponding $\alpha$-chloro- $\alpha(, \alpha)$-(di)fluoroketones $3 \mathrm{~d}$,

(E)- $N$-(2-Chloro-2-fluoro-1-(4-methoxyphenyl)ethylidene)isopropylamine $3 \mathrm{~d}$. The synthesis of imine $\mathbf{3 d}$ is given as a representative example for the synthesis of both imines $\mathbf{3 e}$ and 9a-d. To a cooled $\left(0{ }^{\circ} \mathrm{C}\right)$ solution of $2.30 \mathrm{~g}$ of 2-chloro-2-fluoro-1-(4methoxyphenyl)ethanone $2 \mathrm{~d}(16.3 \mathrm{mmol})$ and $4.82 \mathrm{~g}$ of isopropylamine ( $81.5 \mathrm{mmol}, 5$ equiv) in $20 \mathrm{~mL}$ of dry diethyl ether was added $1.86 \mathrm{~g}$ of titanium(IV) chloride (9.8 mmol, 0.6 equiv) in $4 \mathrm{~mL}$ of dry pentane. The mixture was stirred for $15 \mathrm{~h}$ allowing the temperature to rise to room temperature. After completion of the reaction, the mixture was poured in a biphasic mixture of $50 \mathrm{~mL}$ of aqueous $0.5 \mathrm{M} \mathrm{NaOH}$ solution and $15 \mathrm{~mL}$ of diethyl ether. After separation of the organic phase, the aqueous phase was again extracted with $30 \mathrm{~mL}$ of diethyl ether (2 times). The combined organic phases are dried over a mixture of $\mathrm{MgSO}_{4}$ and $\mathrm{K}_{2} \mathrm{CO}_{3}$ (10/1). After evaporation of the solvent in vacuo, the crude imine $\mathbf{3 d}$ was distilled. Bp $122{ }^{\circ} \mathrm{C}$ ( $2 \mathrm{mmHg}$ ), yield 74\%. Colorless liquid. E/Z ratio 100/0. ${ }^{1} \mathbf{H} \mathbf{~ N M R}\left(300 \mathrm{MHz}, \mathrm{CDCl}_{3}\right): \delta 1.10$ $\left(3 \mathrm{H}, \mathrm{d}, \mathrm{J}=6.2 \mathrm{~Hz}, \mathrm{CH}_{3}\right) ; 1.11\left(3 \mathrm{H}, \mathrm{d}, \mathrm{J}=6.2 \mathrm{~Hz}, \mathrm{CH}_{3}\right) ; 3.55(1 \mathrm{H}$, septet, $\mathrm{J}=6.2 \mathrm{~Hz}$, $\left.\mathrm{C} \underline{\mathrm{H}}\left(\mathrm{CH}_{3}\right)_{2}\right) ; 3.84\left(3 \mathrm{H}, \mathrm{s}, \mathrm{CH}_{3} \mathrm{O}\right) ; 6.59(1 \mathrm{H}, \mathrm{d}, \mathrm{J}=50.6 \mathrm{~Hz}, \mathrm{CHClF}) ; 6.90-7.01(2 \mathrm{H}, \mathrm{m}$, $\left.2 \mathrm{xCH}_{\mathrm{ar}}\right) ; 7.15-7.23\left(2 \mathrm{H}, \mathrm{m}, 2 \mathrm{xCH}_{\mathrm{ar}}\right) .{ }^{19} \mathbf{F} \mathbf{N M R}\left(282 \mathrm{MHz}, \mathrm{CDCl}_{3}\right): \delta-136.3(1 \mathrm{~F}, \mathrm{~d}, \mathrm{~J}=$ 50.6Hz). ${ }^{13} \mathbf{C}$ NMR $\left(75 \mathrm{MHz}, \mathrm{CDCl}_{3}\right.$, int. ref. $\left.=77.26 \mathrm{ppm}\right): \delta 23.5\left(\mathrm{CH}_{3}\right) ; 23.6\left(\mathrm{CH}_{3}\right) ; 52.6$ $\left(\underline{\mathrm{CH}}\left(\mathrm{CH}_{3}\right)_{2}\right) ; 55.5\left(\mathrm{CH}_{3} \mathrm{O}\right) ; 101.9(\mathrm{~d}, \mathrm{~J}=248.1 \mathrm{~Hz}, \mathrm{CClF}) ; 114.1\left(2 \mathrm{xCH}_{\mathrm{ar}}\right) ; 123.7\left(\mathrm{C}_{\mathrm{ar}, \text { quat }}\right)$; 
$129.8\left(2 \mathrm{xCH}_{\mathrm{ar}}\right) ; 160.4\left(\mathrm{C}_{\mathrm{ar}, \text { quat }}\right) ; 162.6(\mathrm{~d}, \mathrm{~J}=24.2 \mathrm{~Hz}, \mathrm{C}=\mathrm{N}) . \mathbf{I R}\left(\mathrm{NaCl}, \mathrm{cm}^{-1}\right): v_{\mathrm{C}=\mathrm{N}} 1649 . \mathbf{M S}$ $(\mathrm{ES}+), \mathrm{m} / \mathrm{z}(\%): 244 / 46\left(\mathrm{M}+\mathrm{H}^{+}, 100\right)$. Copies of ${ }^{1} \mathrm{H} \mathrm{NMR}$ and ${ }^{13} \mathrm{C}$ NMR spectra are included in chapter III of the supporting information file.

(E)-N-(2-Bromo-2-fluoro-1-phenylpropylidene)isopropylamine

Recrystallization (diethyl ether-petroleum ether), mp $48{ }^{\circ} \mathrm{C}$, yield $68 \%$. White crystals. E/Z ratio 100/0. ${ }^{1} \mathbf{H}$ NMR $\left(300 \mathrm{MHz}, \mathrm{CDCl}_{3}\right): \delta 1.04\left(3 \mathrm{H}, \mathrm{d}, \mathrm{J}=6.2 \mathrm{~Hz}, \underline{\mathrm{C}}_{3}\left(\mathrm{CH}_{3}\right) \mathrm{CH}\right) ; 1.08(3 \mathrm{H}$, $\left.\mathrm{d}, \mathrm{J}=6.2 \mathrm{~Hz}, \mathrm{CH}_{3}\left(\mathrm{C}_{3}\right) \mathrm{CH}\right) ; 2.37\left(3 \mathrm{H}, \mathrm{d}, \mathrm{J}=19.3 \mathrm{~Hz}, \mathrm{CH}_{3} \mathrm{CBrF}\right) ; 3.34$ (1H, septet, $\mathrm{J}=6.2 \mathrm{~Hz}$, $\left.\mathrm{C} \underline{\mathrm{H}}\left(\mathrm{CH}_{3}\right)_{2}\right)$; 7.21-7.28 $\left(2 \mathrm{H}, \mathrm{m}, 2 \mathrm{xCH}_{\mathrm{ar}}\right) ; 7.37-7.44\left(3 \mathrm{H}, \mathrm{m}, 3 \mathrm{xCH}_{\mathrm{ar}}\right) .{ }^{19} \mathbf{F}$ NMR $(282 \mathrm{MHz}$, $\left.\mathrm{CDCl}_{3}\right): \delta-99.6(1 \mathrm{~F}, \mathrm{~d}, \mathrm{~J}=19.3 \mathrm{~Hz}) .{ }^{13} \mathbf{C} \mathbf{N M R}\left(75 \mathrm{MHz}, \mathrm{CDCl}_{3}\right): \delta 23.0\left(\mathrm{CH}_{3}\left(\mathrm{CH}_{3}\right) \mathrm{CH}\right) ; 23.3$ $\left(\mathrm{CH}_{3}\left(\underline{\mathrm{CH}}_{3}\right) \mathrm{CH}\right) ; 30.5$ (d, 21.9Hz, $\left.\underline{\mathrm{CH}}_{3} \mathrm{CBrF}\right) ; 52.6\left(\left(\mathrm{CH}_{3}\right)_{2} \mathrm{CH}\right) ; 105.7$ (d, J = 255.0Hz, $\left.\mathrm{CBrF}\right)$; $128.1\left(2 \mathrm{xCH}_{\mathrm{ar}}\right) ; 128.4\left(2 \mathrm{xCH}_{\mathrm{ar}}\right) ; 128.7\left(\mathrm{CH}_{\mathrm{ar}}\right) ; 131.1\left(\mathrm{C}_{\mathrm{ar}, \text { quat }}\right) ; 165.2(\mathrm{~d}, \mathrm{~J}=27.7 \mathrm{~Hz}, \mathrm{C}=\mathrm{N}) . \mathbf{I R}$ $\left(\mathrm{KBr}, \mathrm{cm}^{-1}\right): v_{\mathrm{C}=\mathrm{N}} 1649 ; 2970$. GC-MS (EI): m/z(\%): $272\left(\mathrm{M}^{+}, 0.1\right) ; 192$ (6); 146 (49); 104 (100); 77 (6). Copies of ${ }^{1} \mathrm{H}$ NMR and ${ }^{13} \mathrm{C}$ NMR spectra are included in chapter III of the supporting information file.

(E)-N-(1-Chloro-1,1-difluoro-2-octylidene)isopropylamine 9a. Yield 99\%. Yellowish oil. ${ }^{1} \mathbf{H}$ NMR (300MHz, $\left.\mathrm{CDCl}_{3}\right): \delta$ 0.83-0.94 $\left(3 \mathrm{H}, \mathrm{m}, \mathrm{CH}_{3}\left(\mathrm{CH}_{2}\right)_{5}\right) ; 1.19(6 \mathrm{H}, \mathrm{d}, \mathrm{J}=$ $\left.6.2 \mathrm{~Hz},\left(\mathrm{C}_{3}\right)_{2} \mathrm{CH}\right) ; 1.22-1.44\left(6 \mathrm{H}, \mathrm{m},\left(\mathrm{CH}_{2}\right)_{3}\right) ; 1.47-1.62\left(2 \mathrm{H}, \mathrm{m}, \mathrm{CNCH}_{2} \mathrm{C}_{2}\right) ; 2.40(2 \mathrm{H}, \mathrm{t}, \mathrm{J}$ $\left.=8.1 \mathrm{~Hz}, \mathrm{CNCH}_{2}\right) ; 3.85\left(1 \mathrm{H}\right.$, septet, $\left.\mathrm{CH}\left(\mathrm{CH}_{3}\right)_{2}\right) .{ }^{19} \mathbf{F}$ NMR $\left(282 \mathrm{MHz}, \mathrm{CDCl}_{3}\right): \delta-60.2(2 \mathrm{~F}, \mathrm{~s})$.

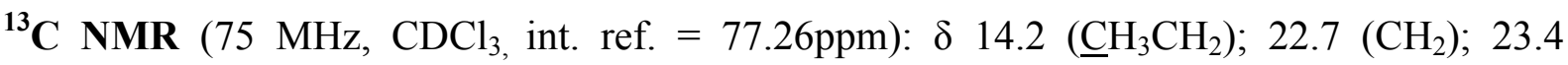
$\left(2 \mathrm{xCH}_{3}\right) ; 27.2\left(\mathrm{CH}_{2}\right) ; 27.7\left(\mathrm{CH}_{2}\right) ; 29.8\left(\mathrm{CH}_{2}\right) ; 31.5\left(\mathrm{CH}_{2}\right) ; 51.4(\mathrm{CH}) ; 124.1(\mathrm{t}, \mathrm{J}=295.4 \mathrm{~Hz}$, $\left.\mathrm{CF}_{2} \mathrm{Cl}\right) ; 160.3(\mathrm{t}, \mathrm{J}=24.8 \mathrm{~Hz}, \mathrm{C}=\mathrm{N}) . \mathbf{I R}\left(\mathrm{NaCl}, \mathrm{cm}^{-1}\right): v_{\mathrm{C}=\mathrm{N}} 1676 ; 1467 . \mathbf{M S}(\mathrm{ES}+), \mathrm{m} / \mathrm{z}(\%)$ : $240 / 42\left(\mathrm{M}+\mathrm{H}^{+}, 100\right)$. Copies of ${ }^{1} \mathrm{H}$ NMR and ${ }^{13} \mathrm{C}$ NMR spectra are included in chapter III of the supporting information file.

(E)- $N$-(1-Chloro-1,1-difluoro-2-decylidene)isopropylamine 9b. Bp 100-110 ${ }^{\circ} \mathrm{C}(6.0$ $\mathrm{mmHg})$, yield 81\%. E/Z ratio 100/0. ${ }^{1} \mathbf{H} \mathbf{N M R}\left(\mathrm{CDCl}_{3}\right): \delta 0.89\left(3 \mathrm{H}, \mathrm{t}, \mathrm{J}=6.7 \mathrm{~Hz}, \mathrm{CH}_{3}\right), 1.19$ $\left(6 \mathrm{H}, \mathrm{d}, \mathrm{J}=6.3 \mathrm{~Hz}, 2 \times \mathrm{CH}_{3}\right), 1.24-1.41\left(10 \mathrm{H}, \mathrm{m}, 5 \times \mathrm{CH}_{2}\right), 1.49-1.60\left(2 \mathrm{H}, \mathrm{m}, \mathrm{CH}_{2}\right), 2.37-2.43$ $\left(2 \mathrm{H}, \mathrm{m}, \mathrm{CH}_{2}\right), 3.81(1 \mathrm{H}$, sept, $\mathrm{J}=6.3 \mathrm{~Hz}, \mathrm{NCH}) .{ }^{19} \mathbf{F}$ NMR $\left(\mathrm{CDCl}_{3}\right): \delta-60.2\left(2 \mathrm{~F}, \mathrm{~s}, \mathrm{CF}_{2} \mathrm{Cl}\right)$. ${ }^{13} \mathbf{C}$ NMR $\left(\mathrm{CDCl}_{3}\right): \delta 14.1\left(\mathrm{CH}_{3}\right), 22.7\left(\mathrm{CH}_{2}\right), 2 \times 23.2\left(2 \times \mathrm{CH}_{3}\right), 27.0\left(\mathrm{CH}_{2}\right), 27.6\left(\mathrm{CH}_{2}\right)$, $29.1\left(2 \mathrm{xCH}_{2}\right), 30.0\left(\mathrm{CH}_{2}\right), 31.8\left(\mathrm{CH}_{2}\right), 51.2(\mathrm{CH}), 124.0\left(\mathrm{t}, \mathrm{J}=295.9 \mathrm{~Hz}, \mathrm{CF}_{2}\right), 160.1(\mathrm{t}, \mathrm{J}=$ 25.4Hz, C=N). IR (NaCl): $v_{\max } 1676 \mathrm{~cm}^{-1}$. MS (ES+), m/z(\%): 268/70 (M+H', 100). Copies of ${ }^{1} \mathrm{H}$ NMR and ${ }^{13} \mathrm{C}$ NMR spectra are included in chapter III of the supporting information file. 
(E)- $\mathrm{N}$-(2-Chloro-2,2-difluoro-1-phenylethylidene)isopropylamine 9c. Mp $42{ }^{\circ} \mathrm{C}$, yield 99\%. E/Z ratio 100/0. White cristals. ${ }^{1} \mathbf{H}$ NMR $\left(300 \mathrm{MHz}, \mathrm{CDCl}_{3}\right): \delta 1.14(6 \mathrm{H}, \mathrm{d}, \mathrm{J}=$ $\left.6.3 \mathrm{~Hz}, 2 \mathrm{xCH}_{3}\right) ; 3.47\left(1 \mathrm{H}\right.$, septet, $\left.\mathrm{J}=6.3 \mathrm{~Hz}, \mathrm{C} \underline{\mathrm{H}}\left(\mathrm{CH}_{3}\right)_{2}\right) ; 7.23-7.26\left(2 \mathrm{H}, \mathrm{m}, 2 \mathrm{xCH}_{\mathrm{ar}}\right) ; 7.43-7.49$ $\left(3 \mathrm{H}, \mathrm{m}, 3 \mathrm{xCH}_{\mathrm{ar}}\right) .{ }^{19} \mathbf{F}$ NMR $\left(282 \mathrm{MHz}, \mathrm{CDCl}_{3}\right): \delta-58.5(2 \mathrm{~F}, \mathrm{~s}) .{ }^{13} \mathbf{C} \mathbf{N M R}\left(75 \mathrm{MHz}, \mathrm{CDCl}_{3}\right.$, int. ref. $=77.26 \mathrm{ppm}): \delta 23.3\left(2 \mathrm{xCH}_{3}\right) ; 53.4\left(\left(\mathrm{CH}_{3}\right)_{2} \mathrm{CH}\right) ; 123.6\left(\mathrm{t}, \mathrm{J}=294.8 \mathrm{~Hz}, \mathrm{CF}_{2} \mathrm{Cl}\right) ; 128.2$ $\left(2 \mathrm{xCH}_{\mathrm{ar}}\right) ; 128.9\left(2 \mathrm{xCH}_{\mathrm{ar}}\right) ; 122.9\left(\mathrm{CH}_{\mathrm{ar}}\right) ; 131.2\left(\mathrm{C}_{\mathrm{ar}, q u a t}\right) ; 159.3(\mathrm{t}, \mathrm{J}=27.1 \mathrm{~Hz}, \mathrm{C}=\mathrm{N}) . \mathbf{I R}(\mathrm{KBr}$,

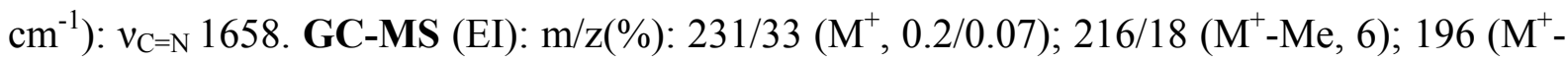
$\mathrm{Cl}, 5) ; 146\left(\mathrm{M}^{+}-\mathrm{CF}_{2} \mathrm{Cl}, 35\right) ; 105$ (9); 104 (100); 103 (8); 77 (11). Copies of ${ }^{1} \mathrm{H}$ NMR and ${ }^{13} \mathrm{C}$ NMR spectra are included in chapter III of the supporting information file.

(E)- $N$-(2,2-Difluoro-1-phenylethylidene)propylamine 9d. Yield 93\%. E/Z ratio 100/0. Yellowish oil. ${ }^{1} \mathbf{H}$ NMR (300MHz, $\left.\mathrm{CDCl}_{3}\right): \delta 0.87\left(3 \mathrm{H}, \mathrm{t}, \mathrm{J}=7.2 \mathrm{~Hz}, \mathrm{CH}_{3}\right) ; 1.67(2 \mathrm{H}$, sextet, $\left.\mathrm{J}=7.2 \mathrm{~Hz}, \mathrm{CH}_{3} \mathrm{CH}_{2}\right) 3.30\left(1 \mathrm{H}, \mathrm{tt}, \mathrm{J}_{\mathrm{HH}}=7.2 \mathrm{~Hz}, \mathrm{~J}_{\mathrm{HF}}=1.7 \mathrm{~Hz}, \mathrm{CH}_{2} \mathrm{~N}\right) ; 7.23-7.27(2 \mathrm{H}, \mathrm{m}$, $\left.2 \mathrm{xCH}_{\mathrm{ar}}\right) ; 7.44-7.48\left(3 \mathrm{H}, \mathrm{m}, 3 \mathrm{xCH}_{\mathrm{ar}}\right) .{ }^{19} \mathbf{F}$ NMR $\left(282 \mathrm{MHz}, \mathrm{CDCl}_{3}\right): \delta-58.0(2 \mathrm{~F}, \mathrm{~s}) .{ }^{13} \mathbf{C} \mathbf{N M R}$ (75 MHz, $\mathrm{CDCl}_{3}$, int. ref. = 77.26ppm): $\delta 11.9\left(\mathrm{CH}_{3}\right) ; 23.3\left(\mathrm{CH}_{3} \underline{\mathrm{CH}}_{2}\right) ; 54.8\left(\mathrm{CH}_{2} \mathrm{~N}\right) ; 123.3(\mathrm{t}$, $\left.\mathrm{J}=294.2 \mathrm{~Hz}, \mathrm{CF}_{2} \mathrm{Cl}\right) ; 127.9\left(2 \mathrm{xCH}_{\mathrm{ar}}\right) ; 128.4\left(2 \mathrm{xCH}_{\mathrm{ar}}\right) ; 129.6\left(\mathrm{CH}_{\mathrm{ar}}\right) ; 130.6\left(\mathrm{C}_{\mathrm{ar}, \text { quat }}\right) ; 160.9(\mathrm{t}, \mathrm{J}$ $=27.1 \mathrm{~Hz}, \mathrm{C}=\mathrm{N}) \cdot \mathbf{I R}\left(\mathrm{NaCl}, \mathrm{cm}^{-1}\right): v_{\mathrm{C}=\mathrm{N}}$ 1663. GC-MS $(\mathrm{EI}): \mathrm{m} / \mathrm{z}(\%): 202\left(\mathrm{M}^{+}-\mathrm{Et}, 3\right) ; 196$ $\left(\mathrm{M}^{+}-\mathrm{Cl}, 6\right) ; 146\left(\mathrm{M}^{+}-\mathrm{CF}_{2} \mathrm{Cl}, 53\right) ; 104$ (100); 77 (14). Copies of ${ }^{1} \mathrm{H}$ NMR and ${ }^{13} \mathrm{C}$ NMR spectra are included in chapter III of the supporting information file.

\subsection{By chlorination or bromination of the corresponding $\alpha(, \alpha)$-(di)fluoroimines} 3a-c, 3f-h, 9e

(E)-N-(2-Chloro-2-fluoro-1-phenylethylidene)isopropylamine 3a. The synthesis of imine 3a is given as a representative example for the synthesis of both imines $\mathbf{3 a - c}, \mathbf{3 f}-\mathbf{h}$ and 12. In a flame dried $100 \mathrm{~mL}$ flask, a heterogeneous mixture of $2.35 \mathrm{~g}$ ( $7.45 \mathrm{mmol}, 1.2$ equiv) of NFSI ( $N$-fluorobenzenesulfonimide) and $1.72 \mathrm{~g}$ (12.42 mmol, 2 equiv) of $\mathrm{K}_{2} \mathrm{CO}_{3}$ and $8 \mathrm{~g}$ of $4 \AA$ molecular sieves (dried at $120^{\circ} \mathrm{C}$ ) in $50 \mathrm{~mL}$ of acetonitrile and $10 \mathrm{~mL}$ of DMF was vigorously stirred under $\mathrm{N}_{2}$ atmosphere for 15 minutes at $0{ }^{\circ} \mathrm{C}$. At the same temperature, 1.00 $\mathrm{g}(6.21 \mathrm{mmol})$ of freshly distilled $N$-(1-phenylethylidene)isopropylamine $\mathbf{1}\left(\mathrm{R}^{1}=\mathrm{H}, \mathrm{R}^{2}=i \mathrm{Pr}\right)$ was added dropwise via a syringe. The mixture was stirred for 2.5 hours at $0{ }^{\circ} \mathrm{C}$. Subsequently, an excess of triethylamine $(5 \mathrm{~mL})$ was added at $0{ }^{\circ} \mathrm{C}$ and stirring was continued for 2 minutes. The reaction mixture was filtered over Celite and the solids were 
washed 3 times with $20 \mathrm{~mL}$ of diethyl ether. Subsequently, the filtrate was poured in $100 \mathrm{~mL}$ of aqueous $0.5 \mathrm{M} \mathrm{NaOH}$. After separation of the organic layer, the aqueous phase was extracted with $3 \times 25 \mathrm{~mL}$ of diethyl ether. To remove the DMF, the combined organic phases were washed with a saturated aqueous $\mathrm{NaCl}$-solution $(2 \times 100 \mathrm{~mL})$ and subsequently dried over $\mathrm{MgSO}_{4} / \mathrm{K}_{2} \mathrm{CO}_{3}(10 / 1)$. Filtration of the drying agents and evaporation of the solvents in vacuo yielded virtually pure $0.64 \mathrm{~g}$ of $\mathrm{N}$-(2-fluoro-1-phenylethylidene)isopropylamine.

Subsequently, to a solution of $0.47 \mathrm{~g}$ of $\mathrm{N}$-(2-fluoro-1-phenylethylidene)isopropylamine (2.6 mmol) in $15 \mathrm{~mL}$ of $\mathrm{CCl}_{4}$ (dry), was added $0.37 \mathrm{~g}$ of $\mathrm{NCS}$ (2.8 mmol, 1.05 equiv). The mixture, fitted with a reflux condenser, was heated with the flame during 2 minutes while swirling. Subsequently, the solution was refluxed during 30 minutes. After cooling down, the mixture was filtered and the filtrate was poured in $30 \mathrm{~mL}$ of water and washed with brine $(2 \mathrm{x}$ $20 \mathrm{~mL}$ ). Subsequently, the organic phase was dried over $\mathrm{MgSO}_{4}$. Filtration of the drying agents and evaporation of the solvents in vacuo yielded imine $\mathbf{3 a}$, sufficiently pure for further use in the next step. Yield 67\%. Yellowish oil. ${ }^{1} \mathbf{H}$ NMR $\left(300 \mathrm{MHz}, \mathrm{CDCl}_{3}\right): \delta 1.10(3 \mathrm{H}, \mathrm{d}, \mathrm{J}$ $\left.=6.2 \mathrm{~Hz}, \mathrm{CH}_{3}\right) ; 1.12\left(3 \mathrm{H}, \mathrm{d}, \mathrm{J}=6.2 \mathrm{~Hz}, \mathrm{CH}_{3}\right) ; 3.49\left(1 \mathrm{H}\right.$, septet, $\left.\mathrm{J}=6.2 \mathrm{~Hz}, \mathrm{C} \underline{\mathrm{H}}\left(\mathrm{CH}_{3}\right)_{2}\right) ; 6.61$ $(1 \mathrm{H}, \mathrm{d}, \mathrm{J}=51.0 \mathrm{~Hz}, \mathrm{CHClF}) ; 7.17-7.26\left(2 \mathrm{H}, \mathrm{m}, 2 \mathrm{xCH}_{\mathrm{ar}}\right) ; 7.41-7.47\left(3 \mathrm{H}, \mathrm{m}, 3 \mathrm{xCH}_{\mathrm{ar}}\right){ }^{{ }^{19} \mathbf{F}}$ NMR $\left(282 \mathrm{MHz}, \mathrm{CDCl}_{3}\right): \delta-136.6(1 \mathrm{~F}, \mathrm{~d}, \mathrm{~J}=51.0 \mathrm{~Hz}) .{ }^{13} \mathbf{C} \mathbf{N M R}\left(75 \mathrm{MHz}, \mathrm{CDCl}_{3}\right.$, int. ref. $=$ 77.26ppm): $\delta 23.4\left(\mathrm{CH}_{3}\right) ; 23.5\left(\mathrm{CH}_{3}\right) ; 52.7\left(\underline{\mathrm{CH}}\left(\mathrm{CH}_{3}\right)_{2}\right) ; 101.6(\mathrm{~d}, \mathrm{~J}=246.9 \mathrm{~Hz}, \mathrm{CHClF})$; $128.3\left(2 \mathrm{xCH}_{\mathrm{ar}}\right) ; 128.6\left(2 \mathrm{xCH}_{\mathrm{ar}}\right) ; 129.4\left(\mathrm{CH}_{\mathrm{ar}}\right) ; 131.6\left(\mathrm{C}_{\mathrm{ar}, \text { quat }}\right) ; 162.5(\mathrm{~d}, \mathrm{~J}=25.4 \mathrm{~Hz}, \mathrm{C}=\mathrm{N}) . \mathbf{I R}$ $\left(\mathrm{NaCl}, \mathrm{cm}^{-1}\right): v_{\mathrm{C}=\mathrm{N}}$ 1651. MS $(\mathrm{ES}+), \mathrm{m} / \mathrm{z}(\%): 213 / 15\left(\mathrm{M}+\mathrm{H}^{+}, 100\right)$. Copies of ${ }^{1} \mathrm{H}$ NMR and ${ }^{13} \mathrm{C}$ NMR spectra are included in chapter III of the supporting information file.

$N$-Butyl- $N$-(2-chloro-2-fluoro-1-phenylethylidene)amine 3b. Yield $81 \%$. ${ }^{\mathbf{1}} \mathbf{H}$ NMR $\left(\mathrm{CDCl}_{3}\right): \delta 0.86\left(3 \mathrm{H}, \mathrm{t}, \mathrm{J}=7.4 \mathrm{~Hz}, \mathrm{CH}_{3}\right), 1.28\left(2 \mathrm{H}, \mathrm{sext}, \mathrm{J}=7.4 \mathrm{~Hz}, \mathrm{CH}_{2}\right), 1.59(2 \mathrm{H}$, quint, $\mathrm{J}=$ 7.4Hz, $\left.\mathrm{CH}_{2}\right), 3.29\left(2 \mathrm{H}, \mathrm{td}, \mathrm{J}=7.4 \mathrm{~Hz}, 2.2 \mathrm{~Hz}, \mathrm{NCH}_{2}\right), 6.63(1 \mathrm{H}, \mathrm{d}, \mathrm{J}=51.1 \mathrm{~Hz}, \mathrm{CHF}), 7.23-$ $7.28\left(2 \mathrm{H}, \mathrm{m}, 2 \times \mathrm{CH}_{\mathrm{ar}}\right), 7.44-7.47\left(3 \mathrm{H}, \mathrm{m}, 3 \times \mathrm{CH}_{\mathrm{ar}}\right) .{ }^{19} \mathbf{F} \mathbf{N M R}\left(\mathrm{CDCl}_{3}\right): \delta-136.5(1 \mathrm{~F}, \mathrm{~d}, \mathrm{~J}=$ 51.1Hz, CHClF). ${ }^{13} \mathrm{C}$ NMR $\left(\mathrm{CDCl}_{3}\right): \delta 13.9\left(\mathrm{CH}_{3}\right), 20.5\left(\mathrm{CH}_{2}\right), 32.6\left(\mathrm{CH}_{2}\right), 52.9\left(\mathrm{CH}_{2}\right)$, $101.4(\mathrm{~d}, \mathrm{~J}=246.9 \mathrm{~Hz}, \mathrm{CHF}), 128.3\left(2 \times \mathrm{CH}_{\mathrm{ar}}\right), 128.5\left(2 \times \mathrm{CH}_{\mathrm{ar}}\right), 129.5\left(\mathrm{CH}_{\mathrm{ar}}\right), 164.7$ (d, J = $25.4 \mathrm{~Hz}, \mathrm{C}=\mathrm{N})$. IR $(\mathrm{NaCl}): v_{\max } 1712,1653 \mathrm{~cm}^{-1}$. MS (ES+), m/z(\%): 228/30 (M+H', 100). Copies of ${ }^{1} \mathrm{H}$ NMR and ${ }^{13} \mathrm{C}$ NMR spectra are included in chapter III of the supporting information file.

(E)- $N$-[2-Chloro-1-(4-chlorophenyl)-2-fluoroethylidene]isopropylamine 3c. Yield 61\%. Redish oil. ${ }^{1} \mathbf{H}$ NMR $\left(300 \mathrm{MHz}, \mathrm{CDCl}_{3}\right): \delta 1.09\left(3 \mathrm{H}, \mathrm{d}, \mathrm{J}=6.2 \mathrm{~Hz}, \mathrm{CH}_{3}\right) ; 1.11(3 \mathrm{H}, \mathrm{d}, \mathrm{J}$ 
$\left.=6.2 \mathrm{~Hz}, \mathrm{CH}_{3}\right) ; 3.45\left(1 \mathrm{H}\right.$, septet, $\left.\mathrm{J}=6.2 \mathrm{~Hz}, \mathrm{C} \underline{\mathrm{H}}\left(\mathrm{CH}_{3}\right)_{2}\right) ; 6.59(1 \mathrm{H}, \mathrm{d}, \mathrm{J}=50.5 \mathrm{~Hz}, \mathrm{CHClF})$; 7.16-7.21 (2H, m, 2xCH $\left.\mathrm{ar}_{\mathrm{ar}}\right)$; 7.41-7.46 (2H, m, $\left.2 \mathrm{xCH}_{\mathrm{ar}}\right) .{ }^{19} \mathbf{F}$ NMR $\left(282 \mathrm{MHz}, \mathrm{CDCl}_{3}\right): \delta-136.6$ $(1 \mathrm{~F}, \mathrm{~d}, \mathrm{~J}=50.5 \mathrm{~Hz}) .{ }^{13} \mathrm{C}$ NMR $\left(75 \mathrm{MHz}, \mathrm{CDCl}_{3}\right): \delta 23.2\left(\mathrm{CH}_{3}\right) ; 23.3\left(\mathrm{CH}_{3}\right) ; 52.7(\underline{\mathrm{CH}}$ $\left.\left(\mathrm{CH}_{3}\right)_{2}\right) ; 101.3(\mathrm{~d}, \mathrm{~J}=248.1 \mathrm{~Hz}, \mathrm{CHClF}) ; 128.8\left(2 \mathrm{xCH}_{\mathrm{ar}}\right) ; 129.6\left(2 \mathrm{xCH}_{\mathrm{ar}}\right) ; 123.0\left(\mathrm{C}_{\mathrm{ar}} \mathrm{Cl}\right)$; $135.6\left(\mathrm{C}_{\text {ar,quat }}\right) ; 161.4(\mathrm{~d}, \mathrm{~J}=25.4 \mathrm{~Hz}, \mathrm{C}=\mathrm{N})$. IR $\left(\mathrm{NaCl}, \mathrm{cm}^{-1}\right): v_{\mathrm{C}=\mathrm{N}} 1653$. MS $(\mathrm{ES}+), \mathrm{m} / \mathrm{z}(\%)$ : $248 / 250 / 252\left(\mathrm{M}+\mathrm{H}^{+}, 100\right)$. Copies of ${ }^{1} \mathrm{H}$ NMR and ${ }^{13} \mathrm{C}$ NMR spectra are included in chapter III of the supporting information file.

(E)-N-(2,2-Dibromo-2-fluoro-1-phenylethylidene)isopropylamine 3f. The synthesis of imine $\mathbf{3} \mathbf{f}$ is analogous to the synthesis of $\mathbf{3 a}$ whereas 2.05 equiv of NBS were added. The reaction mixture was heated under reflux during $2 \mathrm{~h}$ in $\mathrm{CCl}_{4}$. Flash chromatography (hexane/EtOAc/ $/ \mathrm{Et}_{3} \mathrm{~N}$ 94/4/2, $\mathrm{Rf}=0.31$ ), $\mathrm{mp} 37{ }^{\circ} \mathrm{C}$, yield $34 \%$. Yellowish crystals. E/Z ratio 100/0. ${ }^{1} \mathbf{H}$ NMR $\left(300 \mathrm{MHz}, \mathrm{CDCl}_{3}\right): \delta 1.12\left(6 \mathrm{H}, \mathrm{d}, \mathrm{J}=6.2 \mathrm{~Hz}, 2 \mathrm{xCH}_{3}\right) ; 3.35(1 \mathrm{H}$, septet, $\mathrm{J}=$ $\left.6.2 \mathrm{~Hz}, \mathrm{CH}\left(\mathrm{CH}_{3}\right)_{2}\right) ; 7.30-7.35\left(2 \mathrm{H}, \mathrm{m}, 2 \mathrm{xCH}_{\mathrm{ar}}\right) ; 7.38-7.50\left(3 \mathrm{H}, \mathrm{m}, 3 \mathrm{xCH}_{\mathrm{ar}}\right){ }^{19} \mathbf{F} \mathbf{N M R}$ $\left(282 \mathrm{MHz}, \mathrm{CDCl}_{3}\right): \delta-56.6(1 \mathrm{~F}, \mathrm{~s}) .{ }^{13} \mathrm{C}$ NMR $\left(75 \mathrm{MHz}, \mathrm{CDCl}_{3}\right.$, int. ref. $\left.=77.26 \mathrm{ppm}\right): \delta 23.2$ $\left(2 \mathrm{xCH}_{3}\right) ; 53.6\left(\left(\mathrm{CH}_{3}\right)_{2} \mathrm{CH}\right) ; 94.8\left(\mathrm{~d}, \mathrm{~J}=319.6 \mathrm{~Hz}, \mathrm{CBr}_{2} \mathrm{~F}\right) ; 128.5\left(2 \mathrm{xCH}_{\mathrm{ar}}\right) ; 129.1\left(2 \mathrm{xCH}_{\mathrm{ar}}\right)$; $129.6\left(\mathrm{CH}_{\mathrm{ar}}\right) ; 131.5\left(\mathrm{C}_{\mathrm{ar}, \text { quat }}\right) ; 163.1(\mathrm{~d}, \mathrm{~J}=20.8 \mathrm{~Hz}, \mathrm{C}=\mathrm{N}) . \mathbf{I R}\left(\mathrm{KBr}, \mathrm{cm}^{-1}\right): v_{\mathrm{C}=\mathrm{N}} 1648 . \mathbf{M S}$ $(\mathrm{ES}+), \mathrm{m} / \mathrm{z}(\%): 236 / 38 / 40\left(\mathrm{M}+\mathrm{H}^{+}, 50\right)$. Copies of ${ }^{1} \mathrm{H}$ NMR and ${ }^{13} \mathrm{C}$ NMR spectra are included in chapter III of the supporting information file.

(E)-N-(2-Bromo-2-fluoro-1-phenylethylidene)isopropylamine 3g. The synthesis of imine $\mathbf{3 g}$ is analogous to the synthesis of $\mathbf{3 a}$ whereas 1.05 equiv of NBS were added. The reaction mixture was heated during $1 \mathrm{~h}$ in $\mathrm{CCl}_{4}$. Flash chromatography (hexane/EtOAc/Et $3 \mathrm{~N}$ 94/4:2, Rf $=0.26)$, yield $42 \%$. Yellowish oil. E/Z ratio 100/0. ${ }^{1} \mathbf{H}$ NMR $\left(300 \mathrm{MHz}, \mathrm{CDCl}_{3}\right): \delta$ $1.09\left(3 \mathrm{H}, \mathrm{d}, \mathrm{J}=6.2 \mathrm{~Hz}, \mathrm{CH}_{3}\right) ; 1.10\left(3 \mathrm{H}, \mathrm{d}, \mathrm{J}=6.2 \mathrm{~Hz}, \mathrm{CH}_{3}\right) ; 3.48(1 \mathrm{H}$, septet, $\mathrm{J}=6.2 \mathrm{~Hz}$, $\left.\mathrm{C} \underline{\mathrm{H}}\left(\mathrm{CH}_{3}\right)_{2}\right) ; 6.95(1 \mathrm{H}, \mathrm{d}, \mathrm{J}=51.0 \mathrm{~Hz}, \mathrm{CHBrF}) ; 7.24-7.32\left(2 \mathrm{H}, \mathrm{m}, 2 \mathrm{xCH}_{\mathrm{ar}}\right) ; 7.37-7.49(3 \mathrm{H}, \mathrm{m}$, $\left.3 \mathrm{xCH}_{\mathrm{ar}}\right) .{ }^{19} \mathbf{F}$ NMR $\left(282 \mathrm{MHz}, \mathrm{CDCl}_{3}\right): \delta-140.6(1 \mathrm{~F}, \mathrm{~d}, \mathrm{~J}=51.0 \mathrm{~Hz}) .{ }^{13} \mathbf{C} \mathbf{N M R}(75 \mathrm{MHz}$, $\mathrm{CDCl}_{3}$, int. ref. $\left.=77.26 \mathrm{ppm}\right): \delta 23.4\left(\mathrm{CH}_{3}\right) ; 23.5\left(\mathrm{CH}_{3}\right) ; 52.8\left(\underline{\mathrm{CH}}\left(\mathrm{CH}_{3}\right)_{2}\right) ; 93.6(\mathrm{~d}, \mathrm{~J}=$ $259.6 \mathrm{~Hz}, \mathrm{CHFBr}) ; 128.5\left(2 \mathrm{xCH}_{\mathrm{ar}}\right) ; 128.6\left(2 \mathrm{xCH}_{\mathrm{ar}}\right) ; 129.5\left(\mathrm{CH}_{\mathrm{ar}}\right) ; 131.8\left(\mathrm{C}_{\mathrm{ar}, \text { quat }}\right) ; 163.2(\mathrm{~d}, \mathrm{~J}=$ 23.1Hz, C=N). IR $\left(\mathrm{NaCl}, \mathrm{cm}^{-1}\right): v_{\mathrm{C}=\mathrm{N}}$ 1645. MS $(\mathrm{ES}+), \mathrm{m} / \mathrm{z}(\%): 257 / 59\left(\mathrm{M}+\mathrm{H}^{+}, 80\right)$. Copies of ${ }^{1} \mathrm{H}$ NMR and ${ }^{13} \mathrm{C}$ NMR spectra are included in chapter III of the supporting information file. 
(E)- $N$-(2,2-Dichloro-2-fluoro-1-phenylethylidene)isopropylamine $3 \mathbf{h}$. The synthesis of imine $\mathbf{3 h}$ is analogous to the synthesis of $\mathbf{3 a}$ whereas 2.2 equiv of NCS were added. The reaction mixture was heated during $15 \mathrm{~h}$ in $\mathrm{CCl}_{4}$. Flash chromatography (hexane/EtOAc 95/5, $\left.\mathrm{R}_{\mathrm{f}}=0.53\right)$, mp $51-52{ }^{\circ} \mathrm{C}$, yield $89 \%$. E/Z ratio $100 / 0$. White crystals. ${ }^{1} \mathbf{H}$ NMR $(300 \mathrm{MHz}$, $\left.\mathrm{CDCl}_{3}\right): \delta 1.13\left(6 \mathrm{H}, \mathrm{d}, \mathrm{J}=6.2 \mathrm{~Hz}, 2 \mathrm{xCH}_{3}\right) ; 3.37\left(1 \mathrm{H}\right.$, septet, $\left.\mathrm{J}=6.2 \mathrm{~Hz}, \mathrm{CH}\left(\mathrm{CH}_{3}\right)_{2}\right) ; 7.28-7.47$ $\left(5 \mathrm{H}, \mathrm{m}, 5 \mathrm{xCH}_{\mathrm{ar}}\right) .{ }^{19} \mathbf{F}$ NMR $\left(282 \mathrm{MHz}, \mathrm{CDCl}_{3}\right): \delta-58.7(1 \mathrm{~F}, \mathrm{~s}) .{ }^{13} \mathbf{C} \mathbf{N M R}\left(75 \mathrm{MHz}, \mathrm{CDCl}_{3}\right): \delta$ $23.1(2 \mathrm{x} \mathrm{CH}) ; 53.4\left(\underline{\mathrm{CH}}\left(\mathrm{CH}_{3}\right)_{2}\right) ; 117.4\left(\mathrm{~d}, \mathrm{~J}=300.0 \mathrm{~Hz}, \mathrm{CCl}_{2} \mathrm{~F}\right) ; 128.4\left(2 \mathrm{xCH}_{\mathrm{ar}}\right) ; 128.5$ $\left(2 \mathrm{xCH}_{\mathrm{ar}}\right) ; 129.5\left(\mathrm{CH}_{\mathrm{ar}}\right) ; 131.5\left(\mathrm{C}_{\mathrm{ar}, \text { quat }}\right) ; 161.3(\mathrm{~d}, \mathrm{~J}=24.3 \mathrm{~Hz}, \mathrm{C}=\mathrm{N}) . \mathbf{I R}\left(\mathrm{KBr}, \mathrm{cm}^{-1}\right): v_{\mathrm{C}=\mathrm{N}}$ 1651. MS (ES+), m/z(\%): 248/250/252 $\left(\mathrm{M}+\mathrm{H}^{+}, 100\right)$. Copies of ${ }^{1} \mathrm{H}$ NMR and ${ }^{13} \mathrm{C} \mathrm{NMR}$ spectra are included in chapter III of the supporting information file.

(E)-N-(2-Bromo-2,2-difluoro-1-phenylethylidene)isopropylamine 9e. In a dry flask of $50 \mathrm{~mL}, 1.51 \mathrm{~g}$ of $(E)-N$-(2,2-difluoro-1-phenylethylidene)isopropylamine was dissolved in $10 \mathrm{~mL}$ of $\mathrm{CCl}_{4}$. To this solution, $3.01 \mathrm{~g}$ of NBS (18 mmol, 2.2 equiv) was added, followed by the addition of $0.63 \mathrm{~g}$ of AIBN ( $4 \mathrm{mmol}, 0.5$ equiv) in 3 portions. After $8 \mathrm{~h}$ reflux, the mixture was cooled down, filtered and evaporated. Purification with flash chromatography was followed by Kügelrohr distillation. Bp $110{ }^{\circ} \mathrm{C} \quad(1.5 \mathrm{mmHg})$, flash chromatography (hexane/EtOAc 97/3, $\left.\mathrm{R}_{\mathrm{f}}=0,33\right)$, yield 4\%. White crystals. ${ }^{1} \mathbf{H}$ NMR $\left(300 \mathrm{MHz}, \mathrm{CDCl}_{3}\right): \delta$ $1.14(6 \mathrm{H}, \mathrm{d}, \mathrm{J}=6.2 \mathrm{~Hz}, 2 \mathrm{xCH}) ; 3.46\left(1 \mathrm{H}\right.$, septet, $\left.\mathrm{J}=6.2 \mathrm{~Hz}, \mathrm{C} \underline{\mathrm{H}}\left(\mathrm{CH}_{3}\right)_{2}\right) ; 7.25-7.29(2 \mathrm{H}, \mathrm{m}$, $\left.2 \mathrm{xCH}_{\mathrm{ar}}\right) ; 7.43-7.49\left(3 \mathrm{H}, \mathrm{m}, 3 \mathrm{xCH}_{\mathrm{ar}}\right) .{ }^{19} \mathbf{F}$ NMR $\left(282 \mathrm{MHz}, \mathrm{CDCl}_{3}\right): \delta-53.9(2 \mathrm{~F}, \mathrm{~s}) .{ }^{13} \mathbf{C ~ N M R}$ $\left(75 \mathrm{MHz}, \mathrm{CDCl}_{3}\right.$, int. ref. = 77.26ppm): $\delta 23.3\left(2 \mathrm{xCH}_{3}\right) ; 53.4\left(\left(\mathrm{CH}_{3}\right)_{2} \underline{\mathrm{CH}}\right) ; 117.2(\mathrm{t}, \mathrm{J}=$ 309.2Hz, $\left.\mathrm{CF}_{2} \mathrm{Br}\right) ; 128.5\left(2 \mathrm{xCH}_{\mathrm{ar}}\right) ; 128.8\left(2 \mathrm{xCH}_{\mathrm{ar}}\right) ; 129.9\left(\mathrm{CH}_{\mathrm{ar}}\right) ; 131.0\left(\mathrm{C}_{\mathrm{ar}, \text { quat }}\right) ; 160.3(\mathrm{t}, \mathrm{J}=$

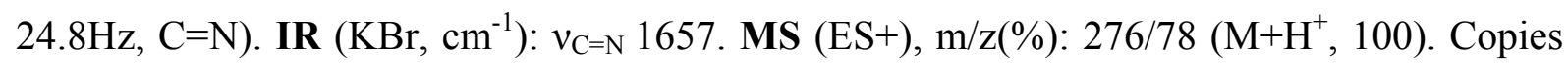
of ${ }^{1} \mathrm{H}$ NMR and ${ }^{13} \mathrm{C}$ NMR spectra are included in chapter III of the supporting information file.

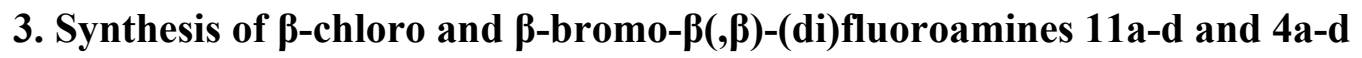

$N$-(2-Chloro-2,2-difluoro-1-phenylethyl)- $N$-propylamine 11d. The synthesis of amine 11d is given as a representative example for the synthesis of both amines 11 and $\mathbf{4 a - d}$. To a solution of $5.86 \mathrm{~g}(25 \mathrm{mmol})$ of imine $9 \mathrm{~d}$ in $150 \mathrm{~mL}$ of methanol was added $1.5 \mathrm{~g}$ of acetic acid (25 mmol, 1.0 equiv) and $1.73 \mathrm{~g}$ of sodium cyanoborohydride (27.5 mmol, 1.1 equiv) at $0{ }^{\circ} \mathrm{C}$. The mixture was stirred for $15 \mathrm{~h}$ while the temperature reached room temperature. After completion of the reaction, the mixture was poured in $150 \mathrm{~mL}$ of a 
saturated aqueous $\mathrm{NaHCO}_{3}$ solution and was subsequently extracted with diethyl ether $(3 \mathrm{x}$ $150 \mathrm{~mL}$ ). The combined organic layers were washed with brine and dried over $\mathrm{MgSO}_{4}$. Filtration of the drying agents and evaporation of the solvents in vacuo yielded amine 11d, sufficiently pure for further use in the next step. Yield 93\%. Colorless oil. ${ }^{1} \mathbf{H}$ NMR $\left(300 \mathrm{MHz}, \mathrm{CDCl}_{3}\right): \delta 0.88\left(3 \mathrm{H}, \mathrm{t}, \mathrm{J}=6.3 \mathrm{~Hz}, \mathrm{CH}_{3}\right) ; 1.41-1.58\left(2 \mathrm{H}, \mathrm{m}, \mathrm{C}_{2} \mathrm{CH}_{3}\right) ; 2.20(1 \mathrm{H}$, $\mathrm{s}(\mathrm{br}), \mathrm{NH}) ; 2.46-2.58\left(2 \mathrm{H}, \mathrm{m}, \mathrm{CH}_{2} \mathrm{~N}\right) ; 4.20\left(1 \mathrm{H}, \mathrm{t}, \mathrm{J}=8.8 \mathrm{~Hz}, \mathrm{CHCClF}_{2}\right) ; 7.34-7.51(5 \mathrm{H}, \mathrm{m}$, $\left.5 \mathrm{XCH}_{\mathrm{ar}}\right) .{ }^{19} \mathrm{~F}$ NMR $\left(282 \mathrm{MHz}, \mathrm{CDCl}_{3}\right): \delta-59.2\left(1 \mathrm{~F}, \mathrm{dd}, \mathrm{J}_{\mathrm{HF}}=8.8 \mathrm{~Hz}, \mathrm{~J}_{\mathrm{FF}}=162.8 \mathrm{~Hz}\right) ;-58.13$ $\left(1 \mathrm{~F}, \mathrm{dd}, \mathrm{J}_{\mathrm{HF}}=9.4 \mathrm{~Hz}, \mathrm{~J}_{\mathrm{FF}}=162.8 \mathrm{~Hz}\right) .{ }^{13} \mathbf{C}$ NMR $\left(300 \mathrm{MHz}\right.$, benzene-d $\mathrm{d}_{6}$, int. ref. $\left.=127.83 \mathrm{ppm}\right)$ : $\delta 11.4\left(\mathrm{CH}_{3}\right) ; 23.1\left(\underline{\mathrm{CH}}_{2} \mathrm{CH}_{3}\right) ; 49.4\left(\mathrm{CH}_{2} \mathrm{~N}\right) ; 70.1\left(\mathrm{t}, \mathrm{J}=24.2 \mathrm{~Hz}, \underline{\mathrm{CCClF}_{2}}\right) ; 128.5\left(2 \mathrm{xCH}_{\mathrm{ar}}\right)$; $128.8\left(\mathrm{CH}_{\mathrm{ar}}\right) ; 129.0\left(2 \mathrm{xCH}_{\mathrm{ar}}\right) ; 130.6\left(\mathrm{t}, \mathrm{J}=297.1 \mathrm{~Hz}, \mathrm{CClF}_{2}\right) ; 135.5\left(\mathrm{C}_{\mathrm{ar}, \text { quat }}\right) \cdot 114.7(\mathrm{dd}, \mathrm{J}=$ $\left.279.2 \mathrm{~Hz}, \mathrm{~J}=289.6 \mathrm{~Hz}, \mathrm{CF}_{2}\right) . \mathbf{I R}\left(\mathrm{NaCl}, \mathrm{cm}^{-1}\right): v_{\mathrm{NH}} 3341 ; 1384 . \mathbf{G C}-\mathbf{M S}(\mathrm{EI}+), \mathrm{m} / \mathrm{z}(\%): 233 / 35$ $\left(\mathrm{M}^{+}, 0.1\right) ; 204 / 06\left(\mathrm{M}^{+}-\mathrm{CH}_{2} \mathrm{CH}_{3}, 9\right) ; 177 / 75\left(\mathrm{M}^{+} \mathrm{NHCH}_{2} \mathrm{CH}_{2} \mathrm{CH}_{3}, 46\right) ; 148\left(\mathrm{M}^{+}-\mathrm{CCl} \mathrm{F} 2,100\right)$; 140(13); 127 (6) 125 (14); 106 (17); 91 (9); 79 (7). Copies of ${ }^{1} \mathrm{H}$ NMR and ${ }^{13} \mathrm{C}$ NMR spectra are included in chapter III of the supporting information file.

$N$-(2-Chloro-2,2-difluoro-1-octyl)- $N$-ispropylamine 11a. Yield 99\%. Yellowish oil. ${ }^{1} \mathbf{H}$ NMR $\left(300 \mathrm{MHz}, \mathrm{CDCl}_{3}\right): \delta 0.79-0.96\left(3 \mathrm{H}, \mathrm{m}, \mathrm{C}_{3} \mathrm{CH}_{2}\right) ; 1.03(3 \mathrm{H}, \mathrm{d}, \mathrm{J}=6.1 \mathrm{~Hz}$, $\left.\mathrm{CH}\left(\mathrm{C}_{3}\right) \mathrm{CH}_{3}\right) ; 1.06\left(3 \mathrm{H}, \mathrm{d}, \mathrm{J}=6,3 \mathrm{~Hz}, \mathrm{CH}\left(\mathrm{CH}_{3}\right) \underline{\mathrm{H}}_{3}\right) ; 1.16-1.44\left(10 \mathrm{H}, \mathrm{m},\left(\mathrm{C}_{2}\right)_{5} \mathrm{CH}_{3}\right) ; 2.93-$ $3.11(2 \mathrm{H}, \mathrm{m}, 2 \mathrm{xCHN}) .{ }^{19} \mathbf{F}$ NMR $\left(282 \mathrm{MHz}, \mathrm{CDCl}_{3}\right): \delta-58.1\left(1 \mathrm{~F}, \mathrm{dd}, \mathrm{J}_{\mathrm{HF}}=6.9 \mathrm{~Hz}, \mathrm{~J}_{\mathrm{FF}}=\right.$ $160.5 \mathrm{~Hz}) ;-57.47\left(1 \mathrm{~F}, \mathrm{dd}, \mathrm{J}_{\mathrm{HF}}=6.5 \mathrm{~Hz}, \mathrm{~J}_{\mathrm{FF}}=160.5 \mathrm{~Hz}\right) .{ }^{13} \mathbf{C ~ N M R}\left(300 \mathrm{MHz}, \mathrm{CDCl}_{3}\right.$, int. ref. $=$ 77.26ppm): $\delta 14.1\left(\mathrm{CH}_{2} \mathrm{CH}_{3}\right) ; 22.6\left(\mathrm{CH}_{2}\right) ; 22.8\left(\mathrm{CH}\left(\underline{\mathrm{CH}}_{3}\right) \mathrm{CH}_{3}\right) ; 24.0\left(\mathrm{CH}_{\left(\mathrm{CH}_{3}\right)} \underline{\mathrm{CH}}_{3}\right) ; 25.8$ $\left(\mathrm{CH}_{2}\right) ; 29.2\left(\mathrm{CH}_{2}\right) ; 29.7\left(\mathrm{~d}, \mathrm{~J}=3.5 \mathrm{~Hz}, \mathrm{CH}_{2}\right) ; 31.7\left(\mathrm{CH}_{2}\right) ; 47.4\left(\underline{\mathrm{CH}}\left(\mathrm{CH}_{3}\right)_{2}\right) ; 62.3(\mathrm{t}, \mathrm{J}=$ $\left.21.9 \mathrm{~Hz}, \underline{\mathrm{CCClF}}_{2}\right) ; 133.0\left(\mathrm{t}, \mathrm{J}=299.9 \mathrm{~Hz}, \mathrm{CClF}_{2}\right) . \mathbf{I R}\left(\mathrm{NaCl}, \mathrm{cm}^{-1}\right): v_{\mathrm{NH}} 3369 ; 2928 ; 1466$; 1101. MS (ES+), m/z(\%): 242/44 (M+H $\left.\mathrm{H}^{+}, 100 / 33\right)$. Copies of ${ }^{1} \mathrm{H} N M R$ and ${ }^{13} \mathrm{C}$ NMR spectra are included in chapter III of the supporting information file.

$N$-(1-Chloro-1,1-difluoro-2-decyl)- $N$-isopropylamine 11b. Flash chromatography (hexane/EtOAc/Et $3 \mathrm{~N}$ 98/1.9/0.1, $\left.\mathrm{R}_{\mathrm{f}}=0.31\right)$, yield 68\%. ${ }^{1} \mathbf{H} \mathbf{N M R}\left(\mathrm{CDCl}_{3}\right): \delta 0.89(3 \mathrm{H}, \mathrm{t}, \mathrm{J}=$ $\left.6.6 \mathrm{~Hz}, \mathrm{CH}_{3}\right), 1.03\left(3 \mathrm{H}, \mathrm{d}, \mathrm{J}=6.3 \mathrm{~Hz}, \mathrm{CH}_{3}\right), 1.06\left(3 \mathrm{H}, \mathrm{d}, \mathrm{J}=6.3 \mathrm{~Hz}, \mathrm{CH}_{3}\right), 1.22-1.41(10 \mathrm{H}, \mathrm{m}$, $\left.5 \mathrm{x} \mathrm{CH}_{2}\right), 1.43-1.59\left(2 \mathrm{H}, \mathrm{m}, \mathrm{CH}_{2}\right), 1.68-1.82\left(2 \mathrm{H}, \mathrm{m}, \mathrm{CH}_{2}\right), 3.04(1 \mathrm{H}$, sept, J = 6.3Hz, $\mathrm{NCH})$.

${ }^{19} \mathbf{F}$ NMR $\left(\mathrm{CDCl}_{3}\right): \delta-57.8\left(2 \mathrm{~F}, \mathrm{~s}, \mathrm{CF}_{2} \mathrm{Cl}\right) .{ }^{13} \mathbf{C} \mathbf{~ N M R}\left(\mathrm{CDCl}_{3}\right): \delta 14.2\left(\mathrm{CH}_{3}\right), 22.7$ and 22.9 $\left(\mathrm{CH}_{3}\right.$ and $\left.2 \times \mathrm{CH}_{2}\right), 24.1\left(\mathrm{CH}_{3}\right), 25.9\left(\mathrm{CH}_{2}\right), 29.3\left(\mathrm{CH}_{2}\right), 29.5\left(\mathrm{CH}_{2}\right), 29.6\left(\mathrm{CH}_{2}\right), 31.4\left(\mathrm{CH}_{2}\right)$, $31.9(\mathrm{CH}), 62.4(\mathrm{t}, \mathrm{J}=21.9 \mathrm{~Hz}, \mathrm{CH}), 133.1\left(\mathrm{t}, \mathrm{J}=300.0 \mathrm{~Hz}, \mathrm{CF}_{2}\right) . \mathbf{I R}(\mathrm{NaCl}): v_{\max } 3370,1466$, $1102 \mathrm{~cm}^{-1}$. MS (ES+), m/z(\%): 270/72 $\left(\mathrm{M}+\mathrm{H}^{+}, 100\right)$. Copies of ${ }^{1} \mathrm{H}$ NMR and ${ }^{13} \mathrm{C}$ NMR spectra are included in chapter III of the supporting information file. 
$N$-(2-Chloro-2,2-difluoro-1-phenylethyl)- $N$-isopropylamine 11c. Yield $99 \%$. Colorless oil. ${ }^{1} \mathbf{H}$ NMR $\left(300 \mathrm{MHz}, \mathrm{CDCl}_{3}\right): \delta 1.04\left(3 \mathrm{H}, \mathrm{d}, \mathrm{J}=6.3 \mathrm{~Hz}, \mathrm{CH}_{3}\right) ; 1.05(3 \mathrm{H}, \mathrm{d}, \mathrm{J}=$ 6,3Hz, $\left.\mathrm{CH}_{3}\right) ; 1.64(1 \mathrm{H}, \mathrm{s}(\mathrm{br}), \mathrm{NH}) ; 2.75\left(1 \mathrm{H}\right.$, septet, $\left.\mathrm{J}=6.3 \mathrm{~Hz}, \mathrm{C} \underline{\mathrm{H}}\left(\mathrm{CH}_{3}\right)_{2}\right) ; 4.27\left(1 \mathrm{H}, \mathrm{t}, \mathrm{J}_{\mathrm{HF}}=\right.$ 9.4Hz, $\left.\mathrm{CHCClF}_{2}\right) ; 7.23-7.43\left(5 \mathrm{H}, \mathrm{m}, 5 \mathrm{xCH}_{\mathrm{ar}}\right) .{ }^{19} \mathbf{F}$ NMR $\left(282 \mathrm{MHz}, \mathrm{CDCl}_{3}\right): \delta-58.2(1 \mathrm{~F}, \mathrm{dd}$, $\left.\mathrm{J}_{\mathrm{HF}}=9.4 \mathrm{~Hz}, \mathrm{~J}_{\mathrm{FF}}=162.1 \mathrm{~Hz}\right) ;-59.4\left(1 \mathrm{~F}, \mathrm{dd}, \mathrm{J}_{\mathrm{HF}}=9.4 \mathrm{~Hz}, \mathrm{~J}_{\mathrm{FF}}=162.1 \mathrm{~Hz}\right) .{ }^{13} \mathbf{C} \mathbf{N M R}(300 \mathrm{MHz}$, $\mathrm{CDCl}_{3}$, int. ref. $\left.=77.26 \mathrm{ppm}\right): \delta 22.0\left(\mathrm{CH}_{3}\right) ; 23.9\left(\mathrm{CH}_{3}\right) ; 46.3\left(\underline{\mathrm{CH}}\left(\mathrm{CH}_{3}\right)_{2}\right) ; 67.5(\mathrm{t}, \mathrm{J}=23.7 \mathrm{~Hz}$, $\left.\mathrm{CCClF}_{2}\right) ; 128.6\left(2 \mathrm{xCH}_{\mathrm{ar}}\right) ; 128.8\left(2 \mathrm{xCH}_{\mathrm{ar}}\right) ; 128.9\left(\mathrm{CH}_{\mathrm{ar}}\right) ; 130.3\left(\mathrm{t}, \mathrm{J}=295.9 \mathrm{~Hz}, \mathrm{CClF}_{2}\right) ; 135.7$ $\left(\mathrm{C}_{\mathrm{ar}, \text { quat }}\right)$. IR $\left(\mathrm{NaCl}, \mathrm{cm}^{-1}\right): v_{\mathrm{NH}} 3341 ; 1455 ; 1384 ; 1074$. MS (ES+), m/z(\%): 234/36 (M+H 100). Copies of ${ }^{1} \mathrm{H}$ NMR and ${ }^{13} \mathrm{C}$ NMR spectra are included in chapter III of the supporting information file.

$N$-(2-Chloro-2-fluoro-1-phenylethyl)- $N$-isopropylamine 4a. Purification via acidbase extraction, yield (mixture of two diastereomers, ratio 58/42) 100\%. ${ }^{1} \mathbf{H}$ NMR $\left(\mathrm{CDCl}_{3}\right)$ : $\delta$

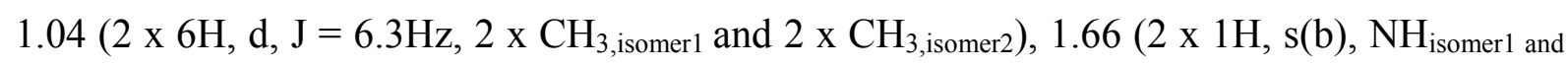

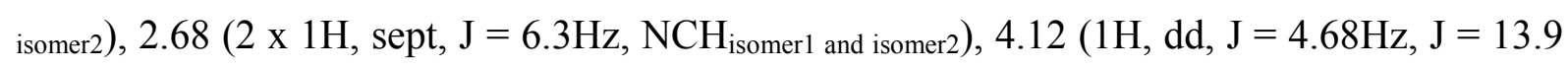
$\left.\mathrm{Hz}, \mathrm{NCH}_{\text {isomer1 }}\right), 4.19\left(1 \mathrm{H}, \mathrm{dd}, \mathrm{J}=5.3 \mathrm{~Hz}, \mathrm{~J}=11.6 \mathrm{~Hz}, \mathrm{NCH}_{\mathrm{isomer} 2}\right), 6.18(2 \mathrm{x} 1 \mathrm{H}, \mathrm{dd}, \mathrm{J}=$

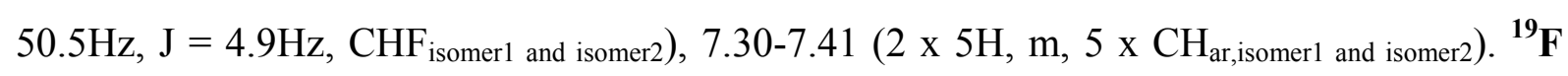
NMR $\left(\mathrm{CDCl}_{3}\right): \delta-138.8\left(1 \mathrm{~F}, \mathrm{dd}, \mathrm{J}=50.5 \mathrm{~Hz}, \mathrm{~J}=11.6 \mathrm{~Hz}, \mathrm{CHF}_{\text {isomer } 2}\right),-138.9(1 \mathrm{~F}, \mathrm{dd}, \mathrm{J}=$

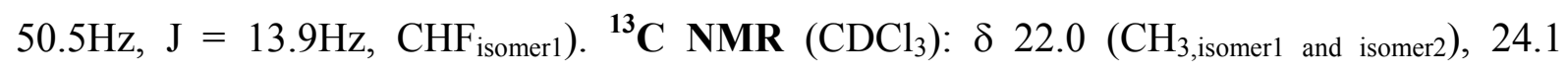

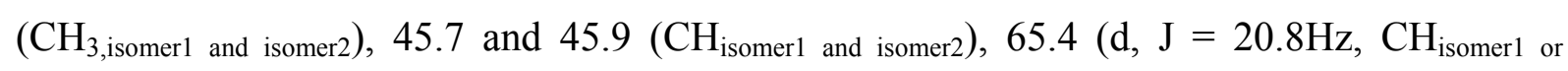

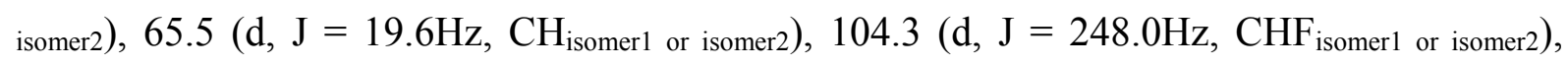

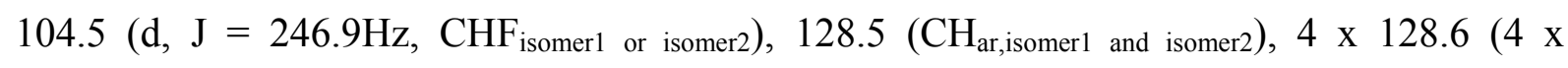
$\left.\mathrm{CH}_{\text {ar,isomer1 and isomer2 }}\right), 137.2\left(\mathrm{C}_{\text {quat,isomer1 and isomer2 }}\right)$ IR $(\mathrm{NaCl}): v_{\max } 3332,1455,1066 \mathrm{~cm}^{-1}$. MS (ES+), m/z(\%): 216/18 (M+H $\left.\mathrm{H}^{+}, 100\right)$. Copies of ${ }^{1} \mathrm{H}$ NMR and ${ }^{13} \mathrm{C}$ NMR spectra are included in chapter III of the supporting information file.

$N$-Butyl- $N$-(2-chloro-2-fluoro-1-phenylethyl)amine 4b. Flash chromatography (hexane/EtOAc/ $\mathrm{Et}_{3} \mathrm{~N}$ 97/2/1, $\mathrm{R}_{\mathrm{f}}=0.30$ ), yield (mixture of two diastereomers, ratio 58:42)

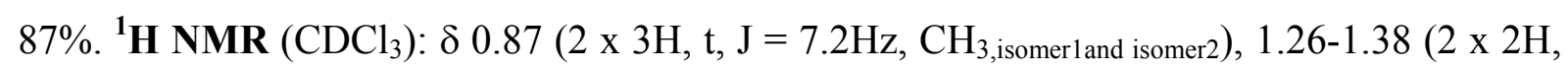

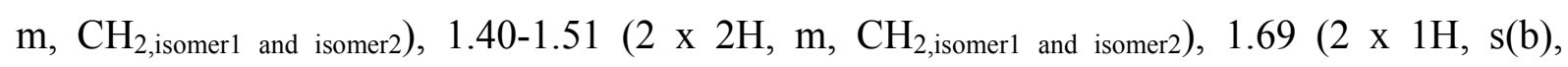
$\mathrm{NH}_{\text {isomer1 and isomer2) }}, 4.02\left(1 \mathrm{H}, \mathrm{dd}, \mathrm{J}=4.8 \mathrm{~Hz}, \mathrm{~J}=12.1 \mathrm{~Hz}, \mathrm{NCH}_{\text {isomer } 1}\right), 4.07(1 \mathrm{H}, \mathrm{dd}, \mathrm{J}=5.7 \mathrm{~Hz}$, $\left.\mathrm{J}=11.4 \mathrm{~Hz}, \mathrm{NCH}_{\text {isomer }}\right), 6.16\left(1 \mathrm{H}, \mathrm{dd}, \mathrm{J}=4.7 \mathrm{~Hz}, \mathrm{~J}=49.0 \mathrm{~Hz}, \mathrm{CHF}_{\text {isomer } 1}\right), 6.17(1 \mathrm{H}, \mathrm{dd}, \mathrm{J}=$ $\left.5.7 \mathrm{~Hz}, \mathrm{~J}=50.5 \mathrm{~Hz}, \mathrm{CHF}_{\text {isomer }}\right), 7.31-7.52\left(2 \times 5 \mathrm{H}, \mathrm{m}, 5 \times \mathrm{CH}_{\text {ar,isomer1 and isomer } 2) .}{ }^{19} \mathbf{F} \mathbf{~ N M R}\right.$ 
$\left(\mathrm{CDCl}_{3}\right): \delta-138.0\left(1 \mathrm{~F}, \mathrm{~d}, \mathrm{~J}=49.0 \mathrm{~Hz}, \mathrm{CHF}_{\text {isomer } 1}\right),-139.2\left(1 \mathrm{~F}, \mathrm{~d}, \mathrm{~J}=50.4 \mathrm{~Hz}, \mathrm{CHF}_{\text {isomer } 2}\right) .{ }^{13} \mathrm{C}$

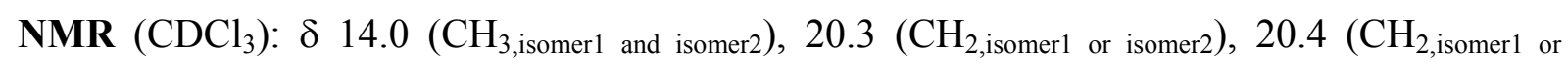

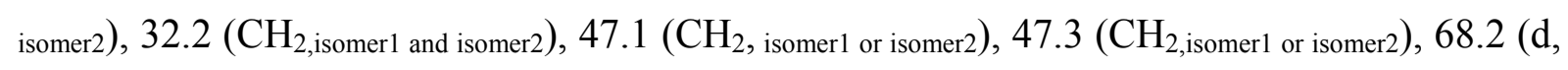

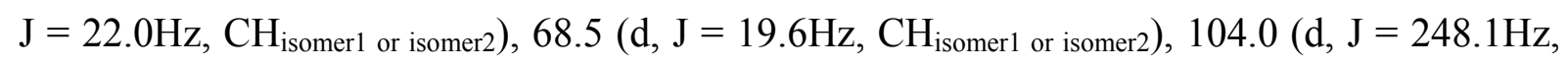

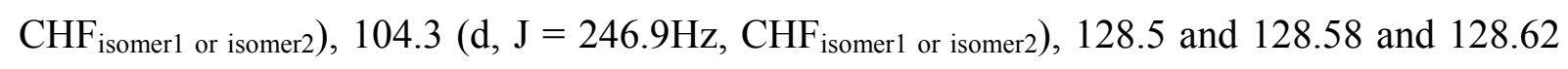

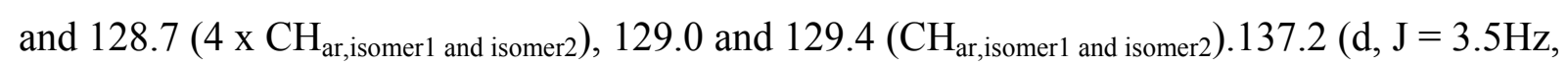
$\mathrm{C}_{\text {quat,isomer1 or isomer2) }}, 137.1\left(\mathrm{~d}, \mathrm{~J}=2.3 \mathrm{~Hz}, \mathrm{C}_{\text {quat isomer1 or isomer2). }} \mathbf{I R}(\mathrm{NaCl}): v_{\max } 3340,1456 \mathrm{~cm}^{-1}\right.$. MS (ES +$), \mathrm{m} / \mathrm{z}(\%): 230\left(\mathrm{M}+\mathrm{H}^{+}, 100\right)$. Anal. Calcd. for $\mathrm{C}_{12} \mathrm{H}_{17} \mathrm{ClFN}: \mathrm{C}, 62.74 ; \mathrm{H}, 7.46 ; \mathrm{N}$, 6.10. Found: C, 62.82; H, 7.75; N, 5.96. Copies of ${ }^{1} \mathrm{H}$ NMR and ${ }^{13} \mathrm{C}$ NMR spectra are included in chapter III of the supporting information file.

$N$-[2-Chloro-1-(4-chlorophenyl)-2-fluoroethyl]- $N$-isopropylamine 4c. Yield $86 \%$. Yellowish oil. ${ }^{1} \mathbf{H}$ NMR $\left(300 \mathrm{MHz}, \mathrm{CDCl}_{3}\right): \delta 1.02\left(3 \mathrm{H}, \mathrm{d}, \mathrm{J}=6.3 \mathrm{~Hz}, \mathrm{CH}_{3}\right) ; 1.03(3 \mathrm{H}, \mathrm{d}, \mathrm{J}=$ $\left.6.3 \mathrm{~Hz}, \mathrm{CH}_{3}\right) ; 1.70(1 \mathrm{H}, \mathrm{s}$, breed, $\mathrm{NH}) ; 2.65\left(1 \mathrm{H}\right.$, septet, $\left.\mathrm{J}=6.3 \mathrm{~Hz}, \mathrm{C} \underline{\mathrm{H}}\left(\mathrm{CH}_{3}\right)_{2}\right) ; 4.10(1 \mathrm{H}$, dd, $\left.\mathrm{J}_{\mathrm{HF}}=14.3 \mathrm{~Hz}, \mathrm{~J}_{\mathrm{HH}}=4.7 \mathrm{~Hz}, \mathrm{C}_{\mathrm{HNH}} \mathrm{isomer1}\right) ; 4.19\left(1 \mathrm{H}, \mathrm{dd}, \mathrm{J}_{\mathrm{HF}}=11.2 \mathrm{~Hz}, \mathrm{~J}_{\mathrm{HH}}=5.2 \mathrm{~Hz}\right.$, $\left.\mathrm{C}_{\mathrm{HNH}} \mathrm{isomer}_{2}\right) ; 6.13\left(1 \mathrm{H}, \mathrm{dd}, \mathrm{J}_{\mathrm{HF}}=50.2 \mathrm{~Hz}, \mathrm{~J}_{\mathrm{HH}}=4.7 \mathrm{~Hz}, \mathrm{CHClF}\right) ; 7.31-7.35\left(4 \mathrm{H}, \mathrm{m}, 4 \mathrm{xCH}_{\mathrm{ar}}\right)$. ${ }^{19}$ F NMR $\left(282 \mathrm{MHz}, \mathrm{CDCl}_{3}\right): \delta-138.7\left(1 \mathrm{~F}, \mathrm{dd}, \mathrm{J}_{\mathrm{HF}}=50.2 \mathrm{~Hz}, \mathrm{~J}_{\mathrm{HF}}=11.2 \mathrm{~Hz}\right.$, isomer 2$) ;-139.8(1 \mathrm{~F}$, $\mathrm{dd}, \mathrm{J}_{\mathrm{HF}}=50.2 \mathrm{~Hz}, \mathrm{~J}_{\mathrm{HF}}=14.3 \mathrm{~Hz}$, isomer1 $) .{ }^{13} \mathbf{C} \mathbf{N M R}\left(75 \mathrm{MHz}, \mathrm{CDCl}_{3}\right): \delta 21.8$ and 21.9

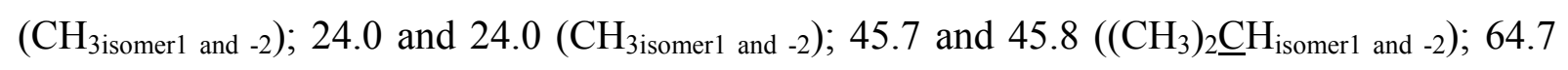
and $64.8(2 \mathrm{xd}, \mathrm{J}=19.6 \mathrm{~Hz}, \underline{\mathrm{C} H C H C l F}$ isomer1 and -2$) ; 103.8\left(\mathrm{~d}, \mathrm{~J}=248.1 \mathrm{~Hz}, \mathrm{CHClF}_{\text {isomer1 of }-2}\right)$; $103.9\left(\mathrm{~d}, \mathrm{~J}=246.9 \mathrm{~Hz}, \mathrm{CHClF}_{\text {isomer1 or }-2)}\right) 128.7\left(2 \mathrm{xCH}_{\text {ar, isomer1 or - } 2)}\right) 128.8\left(2 \mathrm{xCH}_{\text {ar,isomer1 or -2 }}\right)$; $129.9\left(2 \mathrm{xCH}_{\mathrm{ar} \text {,isomer1 and -2 }}\right) ; 130.6$ and $131.2\left(\mathrm{C}_{\mathrm{ar}} \mathrm{Cl}_{\text {isomer1 and }-2}\right) ; 134.3$ and $135.6\left(\mathrm{C}_{\mathrm{ar}, \text { quat,isomerl and }}\right.$ -2). IR $\left(\mathrm{NaCl}, \mathrm{cm}^{-1}\right): v_{\mathrm{NH}} 3334 ; 1492$. MS (ES+), m/z(\%): 250/252/254 (M+H', 100). Copies of ${ }^{1} \mathrm{H}$ NMR and ${ }^{13} \mathrm{C}$ NMR spectra are included in chapter III of the supporting information file.

$\mathrm{N}$-(2-Chloro-2-fluoro-1-(4-methoxyphenyl)ethyl)- $\mathrm{N}$-isopropylamine 4d. Flash chromatography (hexane/EtOAc/ $\mathrm{Et}_{3} \mathrm{~N}$ 97/2/1, Rf $=0.10$ ), yield 82\%. Colorless oil. Ratio major/minor 55/45. ${ }^{1} \mathbf{H}$ NMR $\left(300 \mathrm{MHz}, \mathrm{CDCl}_{3}\right): \delta 1.02\left(6 \mathrm{H}, \mathrm{d}, \mathrm{J}=5.9 \mathrm{~Hz},\left(\mathrm{CH}_{3}\right)_{2} \mathrm{CH}_{(\operatorname{minor})}\right)$; $1.03\left(6 \mathrm{H}, \mathrm{d}, \mathrm{J}=6.6 \mathrm{~Hz},\left(\mathrm{C}_{3}\right)_{2} \mathrm{CH}_{(\text {major })}\right) ; 1.52\left(2 \mathrm{x} 1 \mathrm{H}, \mathrm{s}(\mathrm{br}), \mathrm{NH}_{(\text {major })}\right.$ and $\left.\mathrm{NH}_{(\text {minor })}\right) ; 2.68$ $\left(2 \mathrm{x} 1 \mathrm{H}\right.$, septet, $\mathrm{J}=6.2 \mathrm{~Hz}, \underline{\mathrm{C}}\left(\mathrm{CH}_{3}\right)_{2 \text { (major) }}$ and $\left.\mathrm{C} \underline{\mathrm{H}}\left(\mathrm{CH}_{3}\right)_{2 \text { (minor) }}\right) ; 3.80\left(2 \times 3 \mathrm{H}, \mathrm{s}, \mathrm{CH}_{3} \mathrm{O}_{\text {(major) }}\right.$ and $\left.\mathrm{CH}_{3} \mathrm{O}_{(\text {minor })}\right) ; 4.07\left(1 \mathrm{H}, \mathrm{dd}, \mathrm{J}=14.0 \mathrm{~Hz}, \mathrm{~J}=14.0 \mathrm{~Hz}, \mathrm{CHCFCl}_{(\text {major })}\right) ; 4.14(1 \mathrm{H}, \mathrm{dd}, \mathrm{J}=11.4 \mathrm{~Hz}$, $\left.\mathrm{J}=5.4 \mathrm{~Hz}, \mathrm{CHCFCl}_{(\text {minor })}\right) ; 6.13\left(1 \mathrm{H}, \mathrm{d}, \mathrm{J}=50.4 \mathrm{~Hz}, \mathrm{CHClF}_{(\text {major })}\right) ; 6.15(1 \mathrm{H}, \mathrm{d}, \mathrm{J}=50.1 \mathrm{~Hz}$, $\left.\mathrm{CHClF}_{(\text {minor })}\right)$; 6.86-6.95 (2x2H, 2xm, $2 \mathrm{CH}_{\text {ar(major })}$ and $\left.2 \mathrm{CH}_{\mathrm{ar}(\text { minor })}\right)$; 7.24-7.32 $(2 \mathrm{x} 2 \mathrm{H}, 2 \mathrm{xm}$, 
$2 \mathrm{CH}_{\text {ar(major })}$ and $\left.2 \mathrm{CH}_{\text {ar(minor })}\right){ }^{19} \mathbf{F}$ NMR $\left(282 \mathrm{MHz}, \mathrm{CDCl}_{3}\right): \delta-139.0(1 \mathrm{~F}, \mathrm{dd}, \mathrm{J}=8.5 \mathrm{~Hz}, \mathrm{~J}=$ $\left.49.3 \mathrm{~Hz}, \mathrm{~F}_{\text {(major) }}\right)$; $-138.4\left(1 \mathrm{~F}, \mathrm{~d}, \mathrm{~J}=48.7 \mathrm{~Hz}, \mathrm{~F}_{\text {(minor) }}\right)$. ${ }^{13} \mathbf{C} \mathbf{N M R}\left(75 \mathrm{MHz}, \mathrm{CDCl}_{3}\right.$, int. ref. $=$

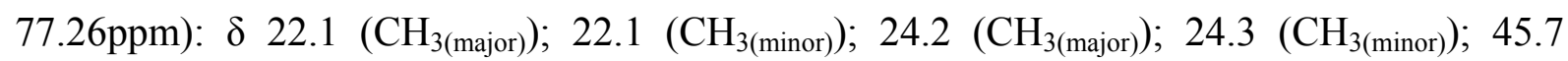
$\left(\left(\mathrm{CH}_{3}\right)_{2} \underline{\mathrm{CH}}_{(\text {major })}\right) ; \quad 45.9 \quad\left(\left(\mathrm{CH}_{3}\right)_{2} \underline{\mathrm{CH}}_{(\text {minor })}\right) ; \quad 55.4 \quad\left(\mathrm{CH}_{3} \mathrm{O}_{\text {(major) }}\right.$ and $\left.\mathrm{CH}_{3} \mathrm{O}_{(\text {minor })}\right) ; \quad 64.8$ $\left(\underline{\mathrm{CHCClF}}_{(\text {minor })}\right) ; 65.1\left(\underline{\mathrm{CHCClF}}_{(\text {major })}\right) ; 104.6\left(\mathrm{~d}, \mathrm{~J}=248.1 \mathrm{~Hz}, \mathrm{CFCl}_{(\text {minor })}\right) ; 104.7$ (d, J = $\left.246.9 \mathrm{~Hz}, \mathrm{CFCl}_{(\text {major })}\right) ; 114.1\left(2 \mathrm{xCH}_{\text {ar(minor })}\right) ; 114.2\left(2 \mathrm{xCH}_{\text {ar(major })}\right) ; 129.2(\mathrm{~d}, \mathrm{~J}=2.3 \mathrm{~Hz}$,

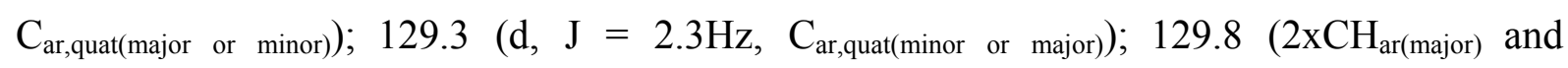
$\left.2 \mathrm{xCH}_{\mathrm{ar}(\text { minor })}\right) ; 159.8\left(\mathrm{C}_{\mathrm{ar}, \text { quat(minor })}\right) ; 159.9\left(\mathrm{C}_{\mathrm{ar} \text {,quat(major })}\right)$. IR $\left(\mathrm{NaCl}, \mathrm{cm}^{-1}\right): v_{\mathrm{NH}} 3369$. MS (ES+), m/z(\%): 246/48 $\left(\mathrm{M}+\mathrm{H}^{+}, 100\right)$. HRMS calcd for $\mathrm{C}_{12} \mathrm{H}_{18} \mathrm{NOClF}\left(\mathrm{M}+\mathrm{H}^{+}\right)$246.10555, found 246.10646. Anal. Calcd. for $\mathrm{C}_{9} \mathrm{H}_{8} \mathrm{FClO}_{2}$ : C, 53.35; H, 3.98; N, 0.00. Found: C, 52.98; H, 4.07; N, 0.26.Copies of ${ }^{1} \mathrm{H}$ NMR and ${ }^{13} \mathrm{C}$ NMR spectra are included in chapter III of the supporting information file.

$N$-(2-Bromo-2-fluoro-1-phenylpropyl)- $N$-isopropylamine 4e. The synthesis of amine $4 \mathbf{e}$ is analogous to the synthesis of $\mathbf{1 1 d}$ whereas the reaction time was 45 minutes and the reaction temperature was $-20{ }^{\circ} \mathrm{C}$. Flash chromatography (hexane/EtOAc/Et $3 \mathrm{~N} \mathrm{96/3/1,} \mathrm{Rf=}$ $0.51)$, yield $45 \%$. Colorless oil. Ratio major/minor 75/25. ${ }^{1} \mathbf{H}$ NMR $\left(300 \mathrm{MHz}, \mathrm{CDCl}_{3}\right): \delta 1.02$ $\left(6 \mathrm{H}, \mathrm{d}, \mathrm{J}=6.3 \mathrm{~Hz},\left(\mathrm{C}_{3}\right)_{2} \mathrm{CH}_{(\text {major })}\right) ; 1.03\left(6 \mathrm{H}, \mathrm{d}, \mathrm{J}=6.3 \mathrm{~Hz},\left(\mathrm{C}_{3}\right)_{2} \mathrm{CH}_{(\text {minor })}\right) ; 2.00(3 \mathrm{H}, \mathrm{d}, \mathrm{J}=$ $\left.20.4 \mathrm{~Hz}, \mathrm{C}_{3}{ }_{3} \mathrm{CBrF}_{\text {(major) }}\right) ; 2.07\left(3 \mathrm{H}, \mathrm{d}, \mathrm{J}=20.4 \mathrm{~Hz}, \mathrm{C}_{3} \mathrm{CBrF}_{\text {(minor })}\right) ; 2.63(2 \times 1 \mathrm{H}$, septet, $\mathrm{J}=$ $6.3 \mathrm{~Hz}, \mathrm{C} \underline{\mathrm{H}}\left(\mathrm{CH}_{3}\right)_{2 \text { (major) }}$ and $\left.\underline{\mathrm{C}}\left(\mathrm{CH}_{3}\right)_{2(\text { minor })}\right) ; 4.04\left(1 \mathrm{H}, \mathrm{d}, \mathrm{J}=13.8 \mathrm{~Hz}, \mathrm{CHCFBr}_{\text {(minor) }}\right) ; 4.11$ $\left(1 \mathrm{H}, \mathrm{d}, \mathrm{J}=11.8 \mathrm{~Hz}, \mathrm{CHCFBr}_{(\text {major })}\right) ; 7.22-7.46\left(2 \mathrm{x} 5 \mathrm{H}, 2 \mathrm{xm}, 5 \mathrm{CH}_{\text {ar(major })}\right.$ and $\left.5 \mathrm{CH}_{\text {ar(minor })}\right) .{ }^{\mathbf{1 9}^{\mathbf{9}} \mathbf{F}}$ NMR $\left(282 \mathrm{MHz}, \mathrm{CDCl}_{3}\right): \delta-100.7\left(1 \mathrm{~F}_{(\text {minor })}\right) ;-96.0\left(1 \mathrm{~F}_{(\text {major })}\right) .{ }^{13} \mathbf{C ~ N M R}\left(300 \mathrm{MHz}, \mathrm{CDCl}_{3}\right.$, int. ref. $=77.26 \mathrm{ppm}): \delta 21.9\left(\underline{\mathrm{CH}_{3}}\left(\mathrm{CH}_{3}\right) \mathrm{CH}_{(\text {major })}\right) ; 21.9 \quad\left(\underline{\mathrm{CH}}_{3}\left(\mathrm{CH}_{3}\right) \mathrm{CH}_{(\text {minor })}\right) ; 24.5$ $\left(\mathrm{CH}_{3}\left(\underline{\mathrm{CH}_{3}}\right) \mathrm{CH}_{\text {(major) }}\right.$ and $\left.\mathrm{CH}_{3}\left(\underline{\mathrm{CH}}_{3}\right) \mathrm{CH}_{(\text {minor })}\right) ; 29.8\left(\mathrm{~d}, \mathrm{~J}=23.1 \mathrm{~Hz}, \mathrm{CH}_{3} \mathrm{CFBr}_{(\text {major })}\right) ; 31.1(\mathrm{~d}, \mathrm{~J}=$ $\left.23.1 \mathrm{~Hz}, \mathrm{CH}_{3} \mathrm{CFBr}_{\text {(minor) }}\right) ; 46.0\left(\underline{\mathrm{CH}}\left(\mathrm{CH}_{3}\right)_{2(\text { minor })}\right) ; 46.2\left(\underline{\mathrm{CH}}\left(\mathrm{CH}_{3}\right)_{2 \text { (major })}\right) ; 70.0(\mathrm{~d}, \mathrm{~J}=20.8 \mathrm{~Hz}$, $\left.\mathrm{CHCFBr}_{\text {(major) }}\right) ; 70.2\left(\mathrm{~d}, \mathrm{~J}=20.8 \mathrm{~Hz}, \mathrm{CHCFBr}_{(\text {minor })}\right) ; 113.2\left(\mathrm{~d}, \mathrm{~J}=259.6 \mathrm{~Hz}, \mathrm{CFBr}_{\text {(minor })}\right) ; 113.8$ $\left(\mathrm{d}, \mathrm{J}=257.3 \mathrm{~Hz}, \mathrm{CFBr}_{(\text {major })}\right) ; 128.4$ and $129.4\left(5 \mathrm{xCH}_{\text {ar(major })}\right.$ and $\left.5 \mathrm{xCH}_{\text {ar(minor })}\right) ; 138.0$ $\left(\mathrm{C}_{\mathrm{ar}, \text { quat(minor })}\right) ; 138.1\left(\mathrm{C}_{\mathrm{ar}, \text { quat(major })}\right)$. IR $\left(\mathrm{NaCl}, \mathrm{cm}^{-1}\right): v_{\mathrm{NH}} 3429 ; v_{\max } 2964,1646$. MS (ES+), $\mathrm{m} / \mathrm{z}(\%): 274 / 76\left(\mathrm{M}+\mathrm{H}^{+}, 100\right)$. Copies of ${ }^{1} \mathrm{H}$ NMR and ${ }^{13} \mathrm{C}$ NMR spectra are included in chapter III of the supporting information file.

\section{Synthesis of monofluoroaziridines 5a-e and 6a-e}

(4-Chlorophenyl)-2-fluoro-1-isopropylaziridine $\mathbf{5 c}$ and $\mathbf{6 c}$. The synthesis of aziridines $\mathbf{5 c}$ and $\mathbf{6 c}$ is given as a representative example for the synthesis of both aziridines $\mathbf{5}$ 
and 6. In a dry $50 \mathrm{~mL}$ flask, $0.33 \mathrm{~g}$ of $\mathrm{K}_{2} \mathrm{CO}_{3}(2.4 \mathrm{mmol}, 1.2$ equiv) was added to a solution of $0.5 \mathrm{~g}$ of $\mathrm{N}$-[2-chloro-1-(4-chlorophenyl)-2-fluoroethyl]- $N$-isopropylamine $4 \mathbf{c}(2 \mathrm{mmol})$ in 20 $\mathrm{mL}$ of DMSO. After refluxing of the solution during $1 \mathrm{~h}$ at $140{ }^{\circ} \mathrm{C}$, the solution was poured in $25 \mathrm{~mL}$ of water and extracted with diethyl ether $(3 \times 25 \mathrm{~mL})$. Drying of the combined organic phases, filtration and evaporation in vacuo, yielded a crude mixture of the two diastereomers, which were separated by flash chromatography. cis-3-(4-Chlorophenyl)-2-fluoro-1isopropylaziridine 5c. Flash chromatography: (hexane/EtOAc 95/5. $\mathrm{R}_{\mathrm{f}}=0.20$ ), yield 36\%. Yellowish oil. ${ }^{1} \mathbf{H}$ NMR $\left(300 \mathrm{MHz}, \mathrm{CDCl}_{3}\right): \delta 1.11(3 \mathrm{H}, \mathrm{d}, \mathrm{J}=6.3 \mathrm{~Hz}, \mathrm{Me}) ; 1.29(3 \mathrm{H}, \mathrm{d}, \mathrm{J}=$ $6.3 \mathrm{~Hz}, \mathrm{Me}) ; 2.04\left(1 \mathrm{H}\right.$, septet $\left.\mathrm{x} \mathrm{d}, \mathrm{J}_{\mathrm{HH}}=6.3 \mathrm{~Hz}, \mathrm{~J}_{\mathrm{HF}}=1.8 \mathrm{~Hz}, \underline{\mathrm{HMe}}_{2}\right) ; 2.54\left(1 \mathrm{H}, \mathrm{dd}, \mathrm{J}_{\mathrm{HH}}=\right.$ $\left.4.4 \mathrm{~Hz}, \mathrm{~J}_{\mathrm{HF}}=2.6 \mathrm{~Hz}, \mathrm{C} \underline{\mathrm{HCHF}}\right) ; 4.77\left(1 \mathrm{H}, \mathrm{dd}, \mathrm{J}_{\mathrm{HF}}=79.1 \mathrm{~Hz}, \mathrm{~J}_{\mathrm{HH}}=4.4 \mathrm{~Hz}, \mathrm{CHF}\right) ; 7.25-7.36(4 \mathrm{H}$, $\left.\mathrm{m}, 4 \mathrm{xCH}_{\mathrm{ar}}\right) .{ }^{19} \mathbf{F} \mathbf{N M R}\left(282 \mathrm{MHz}, \mathrm{CDCl}_{3}\right): \delta-181.62(1 \mathrm{~F}, \mathrm{~d}, \mathrm{~J}=79.1 \mathrm{~Hz}) .{ }^{13} \mathbf{C}$ NMR $(75 \mathrm{MHz}$, $\left.\mathrm{CDCl}_{3}\right): \delta 21.9(\mathrm{Me}) ; 21.3(\mathrm{Me}) ; 44.5(\mathrm{~d}, \mathrm{~J}=13.9 \mathrm{~Hz}, \underline{\mathrm{C} H C H F}) ; 56.8\left(\mathrm{~d}, \mathrm{~J}=2.3 \mathrm{~Hz}, \underline{\mathrm{C}} \mathrm{HMe}_{2}\right)$; $84.1(\mathrm{~d}, \mathrm{~J}=241.1 \mathrm{~Hz}, \mathrm{CHF}) ; 128.3\left(2 \mathrm{xCH}_{\mathrm{ar}}\right) ; 129.2\left(2 \mathrm{xCH}_{\mathrm{ar}}\right) ; 133.2\left(\mathrm{C}_{\mathrm{ar}} \mathrm{Cl}\right) ; 133.6(\mathrm{~d}, \mathrm{~J}=$ 4.6Hz, $\left.\mathrm{C}_{\mathrm{ar} \text {,quat }}\right) . \mathbf{I R}\left(\mathrm{NaCl}, \mathrm{cm}^{-1}\right): v_{\max } 1494 ; 1200 ; 1170 ; 1016 . \mathbf{M S}(\mathrm{ES}+), \mathrm{m} / \mathrm{z}(\%): 214 / 216$ $\left(\mathrm{M}+\mathrm{H}^{+}, 100\right)$. Copies of ${ }^{1} \mathrm{H}$ NMR and ${ }^{13} \mathrm{C}$ NMR spectra are included in chapter III of the supporting information file.

trans-3-(4-Chlorofenyl)-2-fluor-1-isopropylaziridine 6c. Flash chromatography: (hexane/EtOAc 95/5. $\mathrm{R}_{\mathrm{f}}=0.22$ ), yield 28\%. Yellowish oil. ${ }^{1} \mathbf{H}$ NMR $\left(300 \mathrm{MHz}, \mathrm{CDCl}_{3}\right): \delta$ $1.14(3 \mathrm{H}, \mathrm{d}, \mathrm{J}=6.3 \mathrm{~Hz}, \mathrm{Me}) ; 1.20(3 \mathrm{H}, \mathrm{d}, \mathrm{J}=6.3 \mathrm{~Hz}, \mathrm{Me}) ; 2.66(1 \mathrm{H}$, septet, $\mathrm{J}=6.3 \mathrm{~Hz}$, $\left.\mathrm{CHMe}_{2}\right) ; 2.85\left(1 \mathrm{H}, \mathrm{d}, \mathrm{J}_{\mathrm{HF}}=6.1 \mathrm{~Hz}, \mathrm{C} \underline{H C H F}\right) ; 5.00\left(1 \mathrm{H}, \mathrm{d}, \mathrm{J}_{\mathrm{HF}}=79.5 \mathrm{~Hz}, \mathrm{CHF}\right) ; 7.14-7.31$ $\left(4 \mathrm{H}, \mathrm{m}, 4 \mathrm{xCH}_{\mathrm{ar}}\right) .{ }^{\mathbf{1 9}} \mathbf{F}$ NMR $\left(282 \mathrm{MHz}, \mathrm{CDCl}_{3}\right): \delta-185.35(1 \mathrm{~F}, \mathrm{~m}) .{ }^{\mathbf{1 3}} \mathbf{C}$ NMR $(75 \mathrm{MHz}$, $\left.\mathrm{CDCl}_{3}\right): \delta 22.2(\mathrm{Me}) ; 22.3(\mathrm{Me}) ; 43.4(\mathrm{~d}, \mathrm{~J}=16.2 \mathrm{~Hz}, \underline{\mathrm{CHCHF}}) ; 50.1(\mathrm{~d}, \mathrm{~J}=10.4 \mathrm{~Hz}, \underline{\mathrm{CHMe}})_{2}$; $84.4(\mathrm{~d}, \mathrm{~J}=256.1 \mathrm{~Hz}, \mathrm{CHF}) ; 128.4\left(2 \mathrm{xCH}_{\mathrm{ar}}\right) ; 128.5\left(2 \mathrm{xCH}_{\mathrm{ar}}\right) ; 133.3\left(\mathrm{C}_{\mathrm{ar}} \mathrm{Cl}\right) ; 134.6\left(\mathrm{C}_{\mathrm{ar}, \text { quat }}\right)$. IR $\left(\mathrm{NaCl}, \mathrm{cm}^{-1}\right): v_{\max } 1494 ; 1162 ; 1090$. MS (ES+), m/z(\%): 212/214 (100); 214/216 (M+H', 58). Copies of ${ }^{1} \mathrm{H}$ NMR and ${ }^{13} \mathrm{C}$ NMR spectra are included in chapter III of the supporting information file.

cis-2-Fluoro-1-isopropyl-3-phenylaziridine 5a. Flash chromatography (hexane/EtOAc/Et $3 \mathrm{~N}$ 98/1/1, $\left.\mathrm{R}_{\mathrm{f}}=0.09\right)$, yield 31\%. ${ }^{1} \mathbf{H}$ NMR $\left(\mathrm{CDCl}_{3}\right): \delta 1.13(3 \mathrm{H}, \mathrm{d}, \mathrm{J}=$ $\left.6.3 \mathrm{~Hz}, \mathrm{CH}_{3}\right), 1.30\left(3 \mathrm{H}, \mathrm{d}, \mathrm{J}=6.3 \mathrm{~Hz}, \mathrm{CH}_{3}\right), 2.03(1 \mathrm{H}$, sept $\mathrm{x} \mathrm{d}, \mathrm{J}=6.3 \mathrm{~Hz}, \mathrm{~J}=1.9 \mathrm{~Hz}, \mathrm{NCH})$, $2.57(1 \mathrm{H}, \mathrm{dd}, \mathrm{J}=4.4 \mathrm{~Hz}, \mathrm{~J}=2.5 \mathrm{~Hz}, \mathrm{NCH}), 4.77$ ( $1 \mathrm{H}, \mathrm{dd}, \mathrm{J}=79.1 \mathrm{~Hz}, \mathrm{~J}=4.4 \mathrm{~Hz}, \mathrm{CHF}), 7.22-$ $7.42\left(5 \mathrm{H}, \mathrm{m}, 5 \mathrm{x} \mathrm{CH} \mathrm{CH}_{\mathrm{ar}}\right){ }^{19} \mathbf{F}$ NMR $\left(\mathrm{CDCl}_{3}\right): \delta-181.6(1 \mathrm{~F}, \mathrm{~d}(\mathrm{~b}), \mathrm{J}=79.1 \mathrm{~Hz}, \mathrm{CHF}) .{ }^{13} \mathbf{C} \mathbf{N M R}$ $\left(\mathrm{CDCl}_{3}\right): \delta 21.2\left(\mathrm{CH}_{3}\right), 21.5\left(\mathrm{CH}_{3}\right), 45.3(\mathrm{~d}, \mathrm{~J}=13.9 \mathrm{~Hz}, \mathrm{CHCHF}), 56.9(\mathrm{~d}, \mathrm{~J}=3.5 \mathrm{~Hz}, \mathrm{NCH})$, $84.4(\mathrm{~d}, \mathrm{~J}=240.0 \mathrm{~Hz}, \mathrm{CF}), 127.5\left(\mathrm{CH}_{\mathrm{ar}}\right), 128.0\left(2 \times \mathrm{CH}_{\mathrm{ar}}\right), 128.2\left(2 \times \mathrm{CH}_{\mathrm{ar}}\right), 135.1(\mathrm{~d}, \mathrm{~J}=$ 
4.6Hz, $\left.\mathrm{C}_{\text {quat }}\right)$. IR (NaCl): $v_{\max } 1341,1201,1053 \mathrm{~cm}^{-1}$. MS (ES+), m/z(\%): $181\left(\mathrm{M}+2 \mathrm{H}^{+}, 100\right)$. HRMS for $\mathrm{C}_{11} \mathrm{H}_{15} \mathrm{FN}$, Calcd: 180.1183; Found: 180.1186. Anal. Calcd. for $\mathrm{C}_{11} \mathrm{H}_{14} \mathrm{FN}$ : C, 73.71; H, 7.87; N, 7.81. Found: C, 74.02; H, 7.70; N, 7.99. Copies of ${ }^{1} \mathrm{H}$ NMR and ${ }^{13} \mathrm{C}$ NMR spectra are included in chapter III of the supporting information file.

trans-2-Fluoro-1-isopropyl-3-phenylaziridine 6a. Not separated from 5a. ${ }^{1} \mathrm{H},{ }^{13} \mathrm{C}$ and ${ }^{19}$ F NMR spectroscopic data were obtained from the mixture of 5a and $6 \mathbf{6 a} .{ }^{\mathbf{1}} \mathbf{H}$ NMR $\left(\mathrm{CDCl}_{3}\right): \delta 1.11\left(3 \mathrm{H}, \mathrm{d}, \mathrm{J}=6.2 \mathrm{~Hz}, \mathrm{CH}_{3}\right), 1.21\left(3 \mathrm{H}, \mathrm{d}, \mathrm{J}=6.2 \mathrm{~Hz}, \mathrm{CH}_{3}\right), 2.59(1 \mathrm{H}, \mathrm{sept}, \mathrm{J}=$ 6.2Hz, NCH), 2.96 ( 1H, d, J =6.1Hz, NCH), 5.07 ( 1H, d, J = 79.1Hz, CHF), 7.20-7.59 (5H, m, $\left.5 \times \mathrm{CH}_{\mathrm{ar}}\right) .{ }^{19} \mathbf{F} \mathbf{N M R}\left(\mathrm{CDCl}_{3}\right): \delta-181.4$ to $-181.8(1 \mathrm{~F}, \mathrm{~m}, \mathrm{CHF}) .{ }^{13} \mathbf{C ~ N M R}\left(\mathrm{CDCl}_{3}\right): \delta 22.3$ $\left(\mathrm{CH}_{3}\right), 22.4\left(\mathrm{CH}_{3}\right), 44.4(\mathrm{~d}, \mathrm{~J}=16.2 \mathrm{~Hz}, \mathrm{CHCHF}), 50.0(\mathrm{~d}, \mathrm{~J}=10.4 \mathrm{~Hz}, \mathrm{NCH}), 84.5(\mathrm{~d}, \mathrm{~J}=$ 253.8Hz, CF), $127.7\left(\mathrm{CH}_{\mathrm{ar}}\right), 128.2\left(2 \times \mathrm{CH}_{\mathrm{ar}}\right), 128.4\left(2 \times \mathrm{CH}_{\mathrm{ar}}\right), 135.5\left(\mathrm{C}_{\text {quat }}\right)$. Copies of ${ }^{1} \mathrm{H}$ NMR and ${ }^{13} \mathrm{C}$ NMR spectra of the mixture of $\mathbf{5 a}$ and $\mathbf{6 a}$ are included in chapter III of the supporting information file.

cis-1-Butyl-2-fluoro-3-phenylaziridine 5b. Flash chromatography (hexane/EtOAc/Et $3 \mathrm{~N}$ 98/1.9/0.1, $\left.\mathrm{R}_{\mathrm{f}}=0.18\right)$, yield 34\%. ${ }^{1} \mathbf{H} \mathbf{N M R}\left(\mathrm{CDCl}_{3}\right): \delta 0.92(3 \mathrm{H}, \mathrm{t}, \mathrm{J}=$ 7.4Hz, $\left.\mathrm{CH}_{3}\right), 1.36-1.49\left(2 \mathrm{H}, \mathrm{m}, \mathrm{CH}_{2}\right), 1.52-1.68\left(2 \mathrm{H}, \mathrm{m}, \mathrm{CH}_{2}\right), 2.34-2.44$ and 2.72-2.82 (2H, $\left.2 \times \mathrm{m}, \mathrm{NCH}_{2}\right), 2.52(1 \mathrm{H}, \mathrm{dd}, \mathrm{J}=4.3 \mathrm{~Hz}, \mathrm{~J}=2.4 \mathrm{~Hz}, \mathrm{CHCHF}), 4.75(1 \mathrm{H}, \mathrm{dd}, \mathrm{J}=79.2 \mathrm{~Hz}, \mathrm{~J}=$ 4.3Hz, CHF), 7.20-7.40 (5H, m, 5 x CHar $) .{ }^{19} \mathbf{F ~ N M R ~}\left(\mathrm{CDCl}_{3}\right): \delta-182.7(1 \mathrm{~F}, \mathrm{~d}(\mathrm{~b}), \mathrm{J}=79.2 \mathrm{~Hz}$, CHF). ${ }^{13} \mathbf{C}$ NMR $\left(\mathrm{CDCl}_{3}\right): \delta 14.1\left(\mathrm{CH}_{3}\right), 20.4\left(\mathrm{CH}_{2}\right), 31.1\left(\mathrm{CH}_{2}\right), 45.9(\mathrm{~d}, \mathrm{~J}=13.9 \mathrm{~Hz}$, CHCHF), $56.4(\mathrm{~d}, \mathrm{~J}=3.5 \mathrm{~Hz}, \mathrm{NCH}), 85.3(\mathrm{~d}, \mathrm{~J}=241.1 \mathrm{~Hz}, \mathrm{CF}), 127.5\left(\mathrm{CH}_{\mathrm{ar}}\right), 127.8(2 \mathrm{x}$ $\left.\mathrm{CH}_{\mathrm{ar}}\right), 128.2\left(2 \times \mathrm{CH}_{\mathrm{ar}}\right), 134.9\left(\mathrm{~d}, \mathrm{~J}=4.6 \mathrm{~Hz}, \mathrm{C}_{\text {quat }}\right) . \mathbf{I R}(\mathrm{NaCl}): v_{\max } 1456,1200 \mathrm{~cm}^{-1} . \mathbf{M S}$ (ES+), $\mathbf{m} / \mathbf{z}(\%): 194\left(\mathrm{M}+\mathrm{H}^{+}, 100\right)$. Copies of ${ }^{1} \mathrm{H}$ NMR and ${ }^{13} \mathrm{C}$ NMR spectra are included in chapter III of the supporting information file.

trans-1-Butyl-2-fluoro-3-phenylaziridine 6b. Flash chromatography (hexane/EtOAc/Et $3 \mathrm{~N}$ 98/1.9/0.1, $\left.\mathrm{R}_{\mathrm{f}}=0.18\right)$, yield 29\%. ${ }^{1} \mathbf{H} \mathbf{N M R}\left(\mathrm{CDCl}_{3}\right): \delta 0.88(3 \mathrm{H}, \mathrm{t}, \mathrm{J}=$ 7.2Hz, $\left.\mathrm{CH}_{3}\right), 1.25-1.62\left(4 \mathrm{H}, \mathrm{m}, 2 \mathrm{x} \mathrm{CH}_{2}\right), 2.53-2.71\left(2 \mathrm{H}, \mathrm{m}, \mathrm{NCH}_{2}\right), 2.94(1 \mathrm{H}, \mathrm{d}, \mathrm{J}=5.0 \mathrm{~Hz}$, CHCHF), $5.00(1 \mathrm{H}, \mathrm{d}, \mathrm{J}=79.0 \mathrm{~Hz}, \mathrm{CHF}), 7.15-7.47\left(5 \mathrm{H}, \mathrm{m}, 5 \mathrm{x} \mathrm{CH} \mathrm{CH}_{\mathrm{ar}}\right) .{ }^{19} \mathbf{F} \mathbf{N M R}\left(\mathrm{CDCl}_{3}\right): \delta-$ 182.0 to -184.1 (1F, m, CHF). ${ }^{13} \mathbf{C}$ NMR $\left(\mathrm{CDCl}_{3}\right): \delta 14.1\left(\mathrm{CH}_{3}\right), 20.5\left(\mathrm{CH}_{2}\right), 31.9\left(\mathrm{CH}_{2}\right), 44.7$ $(\mathrm{d}, \mathrm{J}=16.2 \mathrm{~Hz}, \mathrm{CHCHF}), 49.0(\mathrm{~d}, \mathrm{~J}=10.4 \mathrm{~Hz}, \mathrm{NCH}), 84.9$ (d, J = 252.7Hz, CF), $127.7\left(\mathrm{CH}_{\mathrm{ar}}\right)$, $128.4\left(2 \times \mathrm{CH}_{\mathrm{ar}}\right), 128.5\left(2 \times \mathrm{CH}_{\mathrm{ar}}\right), 135.9\left(\mathrm{C}_{\text {quat }}\right) . \mathbf{I R}(\mathrm{NaCl}): v_{\max } 1456,1109 \mathrm{~cm}^{-1} . \mathbf{M S}(\mathbf{E S}+)$, 
$\mathbf{m} / \mathbf{z}(\%): 194\left(\mathrm{M}+\mathrm{H}^{+}, 100\right)$. Copies of ${ }^{1} \mathrm{H}$ NMR and ${ }^{13} \mathrm{C}$ NMR spectra of the mixture of $\mathbf{5 b}$ and $\mathbf{6 b}$ are included are included in chapter III of the supporting information file.

Cis-2-Fluoro-1-isopropyl-3-(4-methoxyphenyl)aziridine 5d. The synthesis of aziridine $\mathbf{5 d}$ is analogous to the synthesis of $\mathbf{5 c}$, but the solution was only heated for $0.5 \mathrm{~h}$ at $100{ }^{\circ} \mathrm{C}$. After the reaction only the cis-aziridine was found. Flash chromatography (hexane/EtOAc/Et ${ }_{3} \mathrm{~N}$ 95/4/1, Rf $=0.13$ ), yield $31 \%$. Colorless oil. ${ }^{1} \mathbf{H}$ NMR $(300 \mathrm{MHz}$, $\left.\left.\mathrm{CDCl}_{3}\right): \delta 1.12\left(3 \mathrm{H}, \mathrm{d}, \mathrm{J}=6.1 \mathrm{~Hz}, \mathrm{CH}_{3}\left(\mathrm{C}_{3}\right) \mathrm{CH}\right) ; 1.29\left(3 \mathrm{H}, \mathrm{d}, \mathrm{J}=6.1 \mathrm{~Hz}, \mathrm{C}_{3} \underline{\mathrm{CH}}_{3}\right) \mathrm{CH}\right) ; 2.02$ $\left(1 \mathrm{H}\right.$, septet, $\left.\mathrm{J}=6.1 \mathrm{~Hz}, \mathrm{C} \underline{\mathrm{H}}\left(\mathrm{CH}_{3}\right)_{2}\right) ; 2.53(1 \mathrm{H}, \mathrm{dd}, \mathrm{J}=4.1 \mathrm{~Hz}, \mathrm{~J}=2.3 \mathrm{~Hz}, \mathrm{CHCF}) ; 3.79(3 \mathrm{H}, \mathrm{s}$, $\left.\mathrm{CH}_{3} \mathrm{O}\right) ; 4.74(1 \mathrm{H}, \mathrm{dd}, \mathrm{J}=79.1 \mathrm{~Hz}, \mathrm{~J}=4.1 \mathrm{~Hz}, \mathrm{CHF}) ; 6.87\left(2 \mathrm{H}, \mathrm{d}, \mathrm{J}=8.4 \mathrm{~Hz}, 2 \mathrm{xCH}_{\mathrm{ar}}\right) ; 7.32$ $\left(2 \mathrm{H}, \mathrm{d}, 2 \mathrm{xCH}_{\mathrm{ar}}\right) .{ }^{19} \mathbf{F}$ NMR $\left(282 \mathrm{MHz}, \mathrm{CDCl}_{3}\right): \delta-182.01(1 \mathrm{~F}, \mathrm{~d}, \mathrm{~J}=79.1 \mathrm{~Hz}) .{ }^{13} \mathbf{C}$ NMR $(75$ $\mathrm{MHz}, \mathrm{CDCl}_{3}$, int. ref. = 77.26ppm): $\delta 21.3\left(\underline{\mathrm{CH}}_{3}\left(\mathrm{CH}_{3}\right) \mathrm{CH}\right) ; 21.6\left(\mathrm{CH}_{3}(\underline{\mathrm{CH}} 3) \mathrm{CH}\right) ; 44.9(\mathrm{~d}, \mathrm{~J}=$ 12.7Hz, $\underline{\mathrm{CHCF}}) ; 55.5\left(\mathrm{CH}_{3} \mathrm{O}\right) ; 57.1\left(\mathrm{~d}, \mathrm{~J}=2.3 \mathrm{~Hz},\left(\mathrm{CH}_{3}\right)_{2} \mathrm{CH}\right) ; 84.6(\mathrm{~d}, \mathrm{~J}=240.0 \mathrm{~Hz}, \mathrm{CHF})$; $113.8\left(2 \mathrm{xCH}_{\mathrm{ar}}\right) ; 127.3\left(\mathrm{~d}, \mathrm{~J}=4.6 \mathrm{~Hz}, \underline{\mathrm{C}}_{\text {ar,quat }} \mathrm{CH}\right) ; 129.1\left(2 \mathrm{xCH}_{\mathrm{ar}}\right) ; 159.2\left(\mathrm{C}_{\mathrm{ar}, \text { quat }} \mathrm{O}\right) . \mathbf{I R}(\mathrm{KBr}$, $\left.\mathrm{cm}^{-1}\right): v_{\max } 1516 ; 1614 ; 2967$. MS $(\mathrm{ES}+), \mathrm{m} / \mathrm{z}(\%): 210\left(\mathrm{M}+\mathrm{H}^{+}, 100\right)$. Anal. Calcd. for $\mathrm{C}_{12} \mathrm{H}_{16}$ FNO: C, 68.88; H, 7.71; N, 6.69. Found: C, 68.50; H, 7.93; N, 7.06. Copies of ${ }^{1} \mathrm{H}$ $\mathrm{NMR}$ and ${ }^{13} \mathrm{C}$ NMR spectra are included in chapter III of the supporting information file.

Cis-2-Fluoro-1-isopropyl-2-methyl-3-phenylaziridine 5e. The synthesis of aziridine $\mathbf{5 e}$ is analogous to the synthesis of $\mathbf{5 c}$, but the solution was heated for 40 minutes at $75^{\circ} \mathrm{C}$. Bp

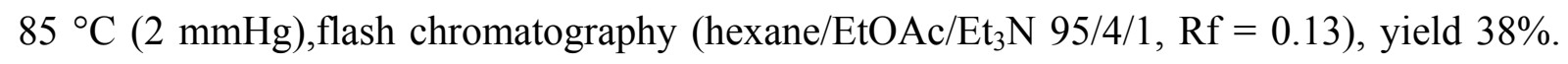
${ }^{1} \mathbf{H}$ NMR $\left(300 \mathrm{MHz}, \mathrm{CDCl}_{3}\right): \delta 1.11\left(3 \mathrm{H}, \mathrm{d}, \mathrm{J}=6.3 \mathrm{~Hz}, \mathrm{CH}_{3}\left(\mathrm{C}_{3}\right) \mathrm{CH}\right) ; 1.28(3 \mathrm{H}, \mathrm{d}, \mathrm{J}=6.1 \mathrm{~Hz}$, $\left.\mathrm{C}_{3}\left(\mathrm{CH}_{3}\right) \mathrm{CH}\right) ; 1.73\left(3 \mathrm{H}, \mathrm{d}, \mathrm{J}=17.6 \mathrm{~Hz}, \mathrm{CH}_{3} \mathrm{CF}\right) ; 2.23\left(1 \mathrm{H}\right.$, septet, $\left.\mathrm{J}=6.2 \mathrm{~Hz}, \mathrm{C} \underline{\mathrm{H}}\left(\mathrm{CH}_{3}\right)_{2}\right)$; $2.36(1 \mathrm{H}, \mathrm{d}, \mathrm{J}=3.3 \mathrm{~Hz}, \mathrm{CHCF}) ; 7.14-7.45\left(5 \mathrm{H}, \mathrm{m}, 5 \mathrm{xCH}_{\mathrm{ar}}\right) .{ }^{19} \mathbf{F} \mathbf{N M R}\left(282 \mathrm{MHz}, \mathrm{CDCl}_{3}\right): \delta-$ $137.22(1 \mathrm{~F}, \mathrm{~d}, \mathrm{~J}=79.1 \mathrm{~Hz}) .{ }^{13} \mathbf{C}$ NMR $\left(75 \mathrm{MHz}, \mathrm{CDCl}_{3}\right.$, int. ref. $\left.=77.26 \mathrm{ppm}\right): \delta 14.9(\mathrm{~d}, \mathrm{~J}=$ $\left.31.2 \mathrm{~Hz}, \mathrm{CH}_{3} \mathrm{CF}\right) ; 22.2\left(\underline{\mathrm{CH}}_{3}\left(\mathrm{CH}_{3}\right) \mathrm{CH}\right) ; 23.0\left(\mathrm{CH}_{3}\left(\underline{\mathrm{CH}}_{3}\right) \mathrm{CH}\right) ; 49.2$ (d, J = 12.7Hz, $\left.\underline{\mathrm{CHCF}}\right) ;$ $54.2\left(\mathrm{~d}, \mathrm{~J}=3.5 \mathrm{~Hz},\left(\mathrm{CH}_{3}\right)_{2} \underline{\mathrm{CH}}\right) ; 88.6(\mathrm{~d}, \mathrm{~J}=233,1 \mathrm{~Hz}, \mathrm{CF}) ; 127.3\left(\mathrm{CH}_{\mathrm{ar}}\right) ; 127.8\left(2 \mathrm{xCH}_{\mathrm{ar}}\right)$; $128.2\left(2 \mathrm{xCH}_{\mathrm{ar}}\right) ; 136.4\left(\mathrm{~d}, \mathrm{~J}=4.6 \mathrm{~Hz}, \mathrm{C}_{\mathrm{ar}, \text { quat }}\right)$. IR $\left(\mathrm{KBr}, \mathrm{cm}^{-1}\right): v_{\max } 1206 ; 1384 ; 2970$. GC-MS (EI): m/z(\%): $193\left(\mathrm{M}^{+}, 3\right) ; 175$ (8); 173 (14); 158 (18); 151 (8); 150 (73); 130 (15); 123 (8); 117 (13); 115 (16); 109 (51); 104 (15); 103 (9); 91 (18); 84 (85); 43 (19); 42 (100); 41 (13). Copies of ${ }^{1} \mathrm{H}$ NMR and ${ }^{13} \mathrm{C}$ NMR spectra are included in chapter III of the supporting information file.

Trans-2-Fluoro-1-isopropyl-2-methyl-3-phenylaziridine 6e. The synthesis of aziridine $\mathbf{6 e}$ is analogous to the synthesis of $\mathbf{6 c}$, but the trans-isomer $\mathbf{6 e}$ was not separable 
from the cis-isomer 5e neither via distillation nor flash chromatography on silica gel. ${ }^{1} \mathbf{H}$ NMR (300MHz, $\left.\mathrm{CDCl}_{3}\right): \delta 1.19\left(3 \mathrm{H}, \mathrm{d}, \mathrm{J}=6.3 \mathrm{~Hz}, \mathrm{CH}_{3}\left(\mathrm{C}_{\underline{3}}\right) \mathrm{CH}\right) ; 1.24(3 \mathrm{H}, \mathrm{d}, \mathrm{J}=6.3 \mathrm{~Hz}$, $\left.\mathrm{C}_{3}\left(\mathrm{CH}_{3}\right) \mathrm{CH}\right) ; 1.39\left(3 \mathrm{H}, \mathrm{d}, \mathrm{J}=18.2 \mathrm{~Hz}, \mathrm{CH}_{3} \mathrm{CF}\right) ; 2.78\left(1 \mathrm{H}\right.$, septet, $\left.\mathrm{J}=6.3 \mathrm{~Hz}, \mathrm{C} \underline{\mathrm{H}}\left(\mathrm{CH}_{3}\right)_{2}\right)$; $2.84(1 \mathrm{H}, \mathrm{d}, \mathrm{J}=9.1 \mathrm{~Hz}, \mathrm{CHCF}) ; 7.18-7.43\left(5 \mathrm{H}, \mathrm{m}, 5 \mathrm{xCH}_{\mathrm{ar}}\right) .{ }^{19} \mathbf{F}$ NMR $\left(282 \mathrm{MHz}, \mathrm{CDCl}_{3}\right): \delta-$ $159.17(1 \mathrm{~F}) .{ }^{13} \mathbf{C ~ N M R}\left(75 \mathrm{MHz}, \mathrm{CDCl}_{3}\right.$, int. ref. $\left.=77.26 \mathrm{ppm}\right): \delta 17.7(\mathrm{~d}, \mathrm{~J}=30.0 \mathrm{~Hz}$, $\left.\mathrm{CH}_{3} \mathrm{CF}\right) ; 22.7\left(\underline{\mathrm{CH}}_{3}\left(\mathrm{CH}_{3}\right) \mathrm{CH}\right) ; 22.9\left(\mathrm{CH}_{3}\left(\underline{\mathrm{CH}}_{3}\right) \mathrm{CH}\right) ; 48.3(\mathrm{~d}, \mathrm{~J}=19.6 \mathrm{~Hz}, \underline{\mathrm{CHCF}}) ; 51.9$ (d, J = $\left.13.9 \mathrm{~Hz},\left(\mathrm{CH}_{3}\right)_{2} \underline{\mathrm{CH}}\right) ; 90.3(\mathrm{~d}, \mathrm{~J}=250.4 \mathrm{~Hz}, \mathrm{CF}) ; 127.2\left(3 \mathrm{xCH}_{\mathrm{ar}}\right) ; 128.4\left(2 \mathrm{xCH}_{\mathrm{ar}}\right) ; 137.0$ $\left(\mathrm{C}_{\mathrm{ar}, \text { quat }}\right)$. IR $\left(\mathrm{KBr}, \mathrm{cm}^{-1}\right): v_{\max } 1206 ; 1384 ; 2970$. GC-MS (EI): m/z(\%): $193\left(\mathrm{M}^{+}, 3\right) ; 175(8)$; 173 (14); 158 (18); 151 (8); 150 (73); 130 (15); 123 (8); 117 (13); 115 (16); 109 (51); 104 (15); 103 (9); 91 (18); 84 (85); 43 (19); 42 (100); 41 (13). Copies of ${ }^{1} \mathrm{H}$ NMR and ${ }^{13} \mathrm{C}$ NMR spectra are included in chapter III of the supporting information file.

\section{Synthesis of 2,2-difluoroaziridines 12a-d}

3-Phenyl-2,2-difluoro-1-isopropylaziridine 12c. In a flame dried $10 \mathrm{~mL}$ flask, a solution of $0.22 \mathrm{~g}$ of diisopropylamine $(2.16 \mathrm{mmol}, 1.3 \mathrm{equiv})$ and $0.86 \mathrm{~mL}$ of butyllithium (2.16 mmol, 1.3 equiv, $2.5 \mathrm{M}$ in hexane) in $5 \mathrm{~mL}$ of THF was stirred under $\mathrm{N}_{2}$ atmosphere for 5 minutes at $-78{ }^{\circ} \mathrm{C}$. At the same temperature, $0.39 \mathrm{~g}$ of $N$-(2-chloro-1-phenyl-2,2difluoroethyl)- $N$-isopropylamine 11c was added dropwise via a syringe. The mixture was stirred for $1 \mathrm{~h}$ at $-78{ }^{\circ} \mathrm{C}$. Subsequently, the mixture was poured in $5 \mathrm{~mL}$ of water. After separation of the organic layer, the aqueous phase was extracted 3 times with diethyl ether. The combined organic layers were dried over $\mathrm{MgSO}_{4}$. Filtration of the drying agent and evaporation of the solvent in vacuo yielded the crude 2,2-difluoroaziridine 12c. Purification was performed with column chromatography on flame dried silica gel (high vacuum) and dry solvents. Flash chromatography: (hexane/EtOAc 98/2. $\mathrm{R}_{\mathrm{f}}=0.23$ ), yield $63 \%$. Colorless oil. ${ }^{1} \mathbf{H}$ NMR (300MHz, $\left.\mathrm{CDCl}_{3}\right): \delta 1.16\left(3 \mathrm{H}, \mathrm{d}, \mathrm{J}=6.3 \mathrm{~Hz}, \mathrm{CH}_{3}\right) ; 1.30\left(3 \mathrm{H}, \mathrm{d}, \mathrm{J}=6.3 \mathrm{~Hz}, \mathrm{CH}_{3}\right) ; 2.69$ $2.79\left(1 \mathrm{H}, \mathrm{m}, \mathrm{CH}\left(\mathrm{CH}_{3}\right)_{2}\right) ; 3.11\left(1 \mathrm{H}, \mathrm{t}, \mathrm{J}=4.1 \mathrm{~Hz}, \mathrm{CHCF}_{2}\right) ; 7.28-7.38\left(5 \mathrm{H}, \mathrm{m}, 5 \mathrm{xCH}_{\mathrm{ar}}\right) .{ }^{{ }^{19} \mathbf{F}}$ NMR $\left(282 \mathrm{MHz}, \mathrm{CDCl}_{3}\right): \delta-130.75\left(1 \mathrm{~F}, \mathrm{dd}, \mathrm{J}_{\mathrm{HF}}=3.5 \mathrm{~Hz}, \mathrm{~J}_{\mathrm{FF}}=95.4 \mathrm{~Hz}\right) ;-116.51\left(1 \mathrm{~F}, \mathrm{dt}, \mathrm{J}_{\mathrm{HF}}=\right.$ $\left.4.3 \mathrm{~Hz}, \mathrm{~J}_{\mathrm{FF}}=95.4 \mathrm{~Hz}\right) .{ }^{13} \mathrm{C}$ NMR $\left(300 \mathrm{MHz}, \mathrm{CDCl}_{3}\right): \delta 21.6\left(\mathrm{CH}_{3}\right) ; 22.7\left(\mathrm{CH}_{3}\right) ; 49.8(\mathrm{dd}, \mathrm{J}=$ $\left.19.6 \mathrm{~Hz}, \mathrm{~J}=16.2 \mathrm{~Hz}, \underline{C C F}_{2}\right) ; 52.5\left(\mathrm{~d}, \mathrm{~J}=9.2 \mathrm{~Hz},\left(\mathrm{CH}_{3}\right)_{2} \mathrm{CH}\right) ; 112.1(\mathrm{dd}, \mathrm{J}=280.4 \mathrm{~Hz}, \mathrm{~J}=$ 293.1Hz, $\left.\mathrm{CF}_{2}\right) ; 127.3\left(2 \mathrm{xCH}_{\mathrm{ar}}\right) ; 128.0\left(\mathrm{CH}_{\mathrm{ar}}\right) ; 128.4\left(2 \mathrm{xCH}_{\mathrm{ar}}\right) ; 133.3$ (d, J = 4.6Hz, C $\mathrm{ar}$,quat). IR $\left(\mathrm{NaCl}, \mathrm{cm}^{-1}\right): v_{\max } 2974 ; 1202$. GC-MS (EI): m/z(\%): $197\left(\mathrm{M}^{+}, 14\right) ; 196\left(\mathrm{M}^{+}-\mathrm{H}, 28\right) ; 182\left(\mathrm{M}^{+}-\right.$ $\left.\mathrm{CH}_{3}, 11\right) ; 155$ (12); $154\left(\mathrm{M}^{+}-\mathrm{CH}\left(\mathrm{CH}_{3}\right)_{2}, 100\right) ; 136$ (12); 127 (92); 109 (7); 77 (7). Copies of ${ }^{1} \mathrm{H}$ NMR and ${ }^{13} \mathrm{C}$ NMR spectra are included in chapter III of the supporting information file. 
3-Phenyl-2,2-difluoro-1-propylaziridine

12d. Flash

chromatography: (hexane/EtOAc 98/2, $\left.\mathrm{R}_{\mathrm{f}}=0.23\right)$, yield 48\%. Yellowish oil. ${ }^{1} \mathbf{H}$ NMR $\left(300 \mathrm{MHz}, \mathrm{CDCl}_{3}\right): \delta$ $0.98\left(3 \mathrm{H}, \mathrm{t}, \mathrm{J}=7.4 \mathrm{~Hz}, \mathrm{CH}_{3}\right) ; 1.64\left(2 \mathrm{H}\right.$, septet, $\left.\mathrm{J}=7.2 \mathrm{~Hz}, \mathrm{CH}_{3} \mathrm{C}_{2}\right) ; 2.65(1 \mathrm{H}, \mathrm{dp}, \mathrm{J}=4.7 \mathrm{~Hz}, \mathrm{~J}$ $=6.4 \mathrm{~Hz}, \mathrm{CH}(\underline{\mathrm{H}}) \mathrm{N}) ; 2.94(1 \mathrm{H}, \mathrm{dp}, \mathrm{J}=3.0 \mathrm{~Hz}, \mathrm{~J}=7.2 \mathrm{~Hz}, \mathrm{C} \underline{\mathrm{H}}(\mathrm{H}) \mathrm{N}) ; 3.06(1 \mathrm{H}, \mathrm{t}, \mathrm{J}=4.0 \mathrm{~Hz}$, $\left.\mathrm{CHCF}_{2}\right) ; 7.29-7.36\left(5 \mathrm{H}, \mathrm{m}, 5 \mathrm{xCH}_{\mathrm{ar}}\right) .{ }^{19} \mathbf{F}$ NMR $\left(282 \mathrm{MHz}, \mathrm{CDCl}_{3}\right): \delta-129.30\left(1 \mathrm{~F}, \mathrm{~d}, \mathrm{~J}_{\mathrm{FF}}=\right.$ 93.4Hz); -118.97 (1F, d, $\left.\mathrm{J}_{\mathrm{FF}}=93.4 \mathrm{~Hz}\right) .{ }^{13} \mathbf{C}$ NMR $\left(300 \mathrm{MHz}, \mathrm{CDCl}_{3}\right): \delta 11.7\left(\mathrm{CH}_{3}\right) ; 22.7$ $\left(\mathrm{CH}_{3} \underline{\mathrm{CH}}_{2}\right) ; 50.9\left(\mathrm{dd}, \mathrm{J}=16.2 \mathrm{~Hz}, \mathrm{~J}=20.8 \mathrm{~Hz}, \underline{C C F}_{2}\right) ; 52.4\left(\mathrm{~d}, \mathrm{~J}=10.4 \mathrm{~Hz}, \mathrm{NCH}_{2}\right) ; 111.9$ (dd, J $\left.=280.4 \mathrm{~Hz}, \mathrm{~J}=293.1 \mathrm{~Hz}, \mathrm{CF}_{2}\right) ; 127.4\left(2 \mathrm{xCH}_{\mathrm{ar}}\right) ; 128.2\left(\mathrm{CH}_{\mathrm{ar}}\right) ; 128.5\left(2 \mathrm{xCH}_{\mathrm{ar}}\right) ; 133.1(\mathrm{~d}, \mathrm{~J}=$ 4.6Hz, $\left.\mathrm{C}_{\mathrm{ar} \text {,quat }}\right)$ IR $\left(\mathrm{NaCl}, \mathrm{cm}^{-1}\right): v_{\max }$ 2966; 1198. GC-MS (EI): m/z(\%): $197\left(\mathrm{M}^{+}, 24\right) ; 196$ $\left(\mathrm{M}^{+}-\mathrm{H}, 77\right) ; 168\left(\mathrm{M}^{+}-\mathrm{CH}_{2} \mathrm{CH}_{3}, 46\right) ; 154\left(\mathrm{M}^{+}-\mathrm{CH}_{2} \mathrm{CH}_{2} \mathrm{CH}_{3}, 79\right) ; 127$ (100); 117 (12); 109 (10); 91(11); 89 (10); 77 (9). Copies of ${ }^{1} \mathrm{H}$ NMR and ${ }^{13} \mathrm{C}$ NMR spectra are included in chapter III of the supporting information file.

2,2-Difluoro-3-hexyl-1-isopropylaziridine 12a. The synthesis of aziridine 12a is analogous to the synthesis of $\mathbf{1 2 c}$, but this aziridine is more stable on silica gel. Flame drying of the silica gel and drying of the solvents is not necessary. Flash chromatography: (hexane/EtOAc 98/2. $\left.\mathrm{R}_{\mathrm{f}}=0.31\right)$, yield $84 \%$. Colorless liquid. ${ }^{1} \mathbf{H}$ NMR $\left(300 \mathrm{MHz}, \mathrm{CDCl}_{3}\right): \delta$ 0.84-0.93 (3H, m, $\left.\underline{\mathrm{C}}_{3}\left(\mathrm{CH}_{2}\right)_{5}\right) ; 1.12\left(3 \mathrm{H}, \mathrm{d}, \mathrm{J}=6.2 \mathrm{~Hz}, \mathrm{CH}\left(\mathrm{CH}_{3}\right) \mathrm{CH}_{3}\right) ; 1.20(3 \mathrm{H}, \mathrm{d}, \mathrm{J}=6.2 \mathrm{~Hz}$, $\left.\mathrm{CH}\left(\mathrm{CH}_{3}\right) \underline{\mathrm{H}}_{3}\right) ; 1.24-1.65\left(10 \mathrm{H}, \mathrm{m},\left(\mathrm{CH}_{2}\right)_{5}\right) ; 1.97-2.06\left(1 \mathrm{H}, \mathrm{m}, \mathrm{CHCF}_{2}\right) ; 2.46(1 \mathrm{H}$, septet, $\mathrm{J}=$ $\left.6.2 \mathrm{~Hz}, \mathrm{C} \underline{\mathrm{H}}\left(\mathrm{CH}_{3}\right)_{2}\right) .{ }^{19} \mathbf{F}$ NMR $\left(282 \mathrm{MHz}, \mathrm{CDCl}_{3}\right): \delta-133.72\left(1 \mathrm{~F}, \mathrm{~d}, \mathrm{~J}_{\mathrm{FF}}=101.3 \mathrm{~Hz}\right) ;-116.49$ $\left(1 \mathrm{~F}, \mathrm{~d}, \mathrm{~J}_{\mathrm{FF}}=101.3 \mathrm{~Hz}\right) \cdot{ }^{13} \mathrm{C} \mathbf{N M R}\left(300 \mathrm{MHz}, \mathrm{CDCl}_{3}\right.$, int. ref. $\left.=77.26 \mathrm{ppm}\right): \delta 14.3$ $\left(\underline{\mathrm{CH}}_{3}\left(\mathrm{CH}_{2}\right)_{5} ; 22.1\left(\mathrm{CH}\left(\underline{\mathrm{CH}}_{3}\right) \mathrm{CH}_{3}\right) ; 22.8\left(\mathrm{CH}_{3} \underline{\mathrm{CH}}_{2}\right) ; 23.0\left(\mathrm{CH}\left(\mathrm{CH}_{3}\right) \underline{\mathrm{CH}_{3}}\right) ; 23.0\left(\mathrm{CH}_{2}\right) ; 27.9(\mathrm{~d}\right.$, $\left.\mathrm{J}=27.8 \mathrm{~Hz}, \mathrm{CH}_{2}\right) ; 29.1\left(\mathrm{CH}_{2}\right) ; 31.9\left(\mathrm{CH}_{2}\right) ; 48.2\left(\mathrm{dd}, \mathrm{J}=15.6 \mathrm{~Hz}, \mathrm{~J}=17.88 \mathrm{~Hz}, \mathrm{CHCF}_{2}\right) ; 52.0$ $\left(\mathrm{d}, \mathrm{J}=10.4 \mathrm{~Hz}, \underline{\mathrm{CH}}\left(\mathrm{CH}_{3}\right)_{2}\right) ; 114.2\left(\mathrm{dd}, \mathrm{J}=279.2 \mathrm{~Hz}, \mathrm{~J}=289.6 \mathrm{~Hz}, \mathrm{CF}_{2}\right) . \mathbf{I R}\left(\mathrm{NaCl}, \mathrm{cm}^{-1}\right): v_{\max }$ 2960; 1469; 1211. GC-MS (EI): m/z(\%): $190\left(\mathrm{M}^{+}-\mathrm{CH}_{3}, 72\right) ; 162\left(\mathrm{M}^{+}-\mathrm{CH}\left(\mathrm{CH}_{3}\right)_{2}, 100\right) ; 148$ (87); 142 (15); 134 (11); 122 (9); 120 (98); 106 (100); 94 (24); 93 (21); 92 (17); 80(11); 78 (10); 77 (17); 69 (14); 55 (65); 43 (43). Anal. Calcd. for $\mathrm{C}_{11} \mathrm{H}_{21} \mathrm{~F}_{2} \mathrm{~N}$ : C, 64.36; H, 10.31; N, 6.82. Found: C, 64.08; H, 10.68; N, 7.11. Copies of ${ }^{1} \mathrm{H}$ NMR and ${ }^{13} \mathrm{C}$ NMR spectra are included in chapter III of the supporting information file.

2,2-Difluoro-1-isopropyl-3-octylaziridine 12b. Flash chromatography (short column) (hexane/EtOAc/Et $3 \mathrm{~N}$ 98/1.9/0.1, $\left.\mathrm{R}_{\mathrm{f}}=0.25\right)$, yield 64\%. ${ }^{1} \mathbf{H} \mathbf{N M R}\left(\mathrm{CDCl}_{3}\right): \delta 0.88(3 \mathrm{H}, \mathrm{t}, \mathrm{J}=$ $\left.6.6 \mathrm{~Hz}, \mathrm{CH}_{3}\right), 1.12\left(3 \mathrm{H}, \mathrm{d}, \mathrm{J}=6.3 \mathrm{~Hz}, \mathrm{CH}_{3}\right), 1.20\left(3 \mathrm{H}, \mathrm{d}, \mathrm{J}=6.3 \mathrm{~Hz}, \mathrm{CH}_{3}\right), 1.23-1.39(10 \mathrm{H}, \mathrm{m}$, 
$\left.5 \times \mathrm{CH}_{2}\right), 1.98-2.06(1 \mathrm{H}, \mathrm{m}, \mathrm{CH}), 2.47(1 \mathrm{H}$, sept $\mathrm{x} \mathrm{d}, \mathrm{J}=6.3 \mathrm{~Hz}, \mathrm{~J}=6.1 \mathrm{~Hz}, \mathrm{NCH}) .{ }^{19} \mathbf{F ~ N M R}$ $\left(\mathrm{CDCl}_{3}\right): \delta-116.5\left(\mathrm{~d}, \mathrm{~J}_{\mathrm{FF}}=98.7 \mathrm{~Hz}, \mathrm{CF}_{a} \mathrm{~F}_{\mathrm{b}}\right),-133.7\left(1 \mathrm{~F}, \mathrm{~d}, \mathrm{~J}_{\mathrm{FF}}=98.7 \mathrm{~Hz}, \mathrm{CF}_{\mathrm{a}} F_{b}\right) .{ }^{13} \mathbf{C} \mathbf{N M R}$ $\left(\mathrm{CDCl}_{3}\right): \delta 14.1\left(\mathrm{CH}_{3}\right), 21.8\left(\mathrm{CH}_{3}\right), 22.7\left(\mathrm{CH}_{2}\right), 22.8\left(\mathrm{CH}_{3}\right), 26.8\left(\mathrm{CH}_{2}\right), 27.7\left(\mathrm{CH}_{2}\right), 2 \times 29.3$ $\left(2 \times \mathrm{CH}_{3}\right), 29.5\left(\mathrm{CH}_{2}\right), 31.9\left(\mathrm{CH}_{2}\right), 48.0\left(\mathrm{dd}, \mathrm{J}=16.2 \mathrm{~Hz}, \mathrm{~J}=18.5 \mathrm{~Hz}, \mathrm{CHCF}_{2}\right), 51.8(\mathrm{~d}, \mathrm{~J}=$ 9.2Hz, NCH), $114.0\left(\mathrm{dd}, \mathrm{J}=279.2 \mathrm{~Hz}, \mathrm{~J}=289.6 \mathrm{~Hz}, \mathrm{CF}_{2}\right) . \mathbf{I R}(\mathrm{NaCl}): v_{\max } 1470,1210 \mathrm{~cm}^{-1}$. MS (ES+), m/z(\%): $234\left(\mathrm{M}+\mathrm{H}^{+}, 100\right)$. Copies of ${ }^{1} \mathrm{H}$ NMR and ${ }^{13} \mathrm{C}$ NMR spectra are included in chapter III of the supporting information file.

\section{Synthesis of amides 15 and 16}

$N$-Isopropyl-2-fluoro-2-phenylacetamide 15a. The synthesis of amide 15a is given as a representative example for the synthesis of amides 15 After one week standing in moist air (refrigerator), difluoroaziridine 12c was degraded to amide 15a. Mp: $68-70{ }^{\circ} \mathrm{C}$. Flash chromatography: (hexane/EtOAc 90/10, $\mathrm{R}_{\mathrm{f}}=0.14$ ), yield $76 \%$. White crystals. ${ }^{1} \mathbf{H}$ NMR $\left(300 \mathrm{MHz}, \mathrm{CDCl}_{3}\right): \delta 1.19\left(3 \mathrm{H}, \mathrm{d}, \mathrm{J}=6.3 \mathrm{~Hz}, \mathrm{CH}\left(\mathrm{C}_{3}\right) \mathrm{CH}_{3}\right) ; 1.23(3 \mathrm{H}, \mathrm{d} \mathrm{J}=6.6 \mathrm{~Hz}$, $\left.\mathrm{CH}\left(\mathrm{CH}_{3}\right) \underline{\mathrm{H}}_{3}\right) ; 4.07-4.23\left(1 \mathrm{H}, \mathrm{m}, \mathrm{C} \underline{\mathrm{H}}\left(\mathrm{CH}_{3}\right)_{2}\right) ; 5.73(1 \mathrm{H}, \mathrm{d}, \mathrm{J}=48.4 \mathrm{~Hz}, \mathrm{CHF}) ; 6.37(1 \mathrm{H}$, $\mathrm{s}(\mathrm{br}), \mathrm{NH}) ; 7.35-7.46\left(5 \mathrm{H}, \mathrm{m}, 5 \mathrm{xCH}_{\mathrm{ar}}\right) .{ }^{19} \mathbf{F}$ NMR $\left(282 \mathrm{MHz}, \mathrm{CDCl}_{3}\right): \delta-175.78(1 \mathrm{~F}, \mathrm{dd}, \mathrm{J}=$ $48.7 \mathrm{~Hz}, \quad \mathrm{~J}=4.0 \mathrm{~Hz}) .{ }^{13} \mathbf{C} \mathbf{N M R}\left(300 \mathrm{MHz}, \mathrm{CDCl}_{3}\right.$, int. ref. $\left.=77.26 \mathrm{ppm}\right): \delta 22.8$ $\left(\mathrm{CH}\left(\underline{\mathrm{CH}}_{3}\right) \mathrm{CH}_{3}\right) ; 22,9\left(\mathrm{CH}\left(\mathrm{CH}_{3}\right) \underline{\mathrm{C}} \mathrm{H}_{3}\right) ; 41.5\left(\underline{\mathrm{CH}}\left(\mathrm{CH}_{3}\right)_{2}\right) ; 92.0(\mathrm{~d}, \mathrm{~J}=186.9 \mathrm{~Hz}, \mathrm{CHF}) ; 126.9$ (d, $\left.\mathrm{J}=5.8 \mathrm{~Hz}, 2 \mathrm{xCH}_{\mathrm{ar}}\right) ; 128.9\left(2 \mathrm{xCH}_{\mathrm{ar}}\right) ; 129.6\left(\mathrm{~d}, \mathrm{~J}=2.3 \mathrm{~Hz}, \mathrm{CH}_{\mathrm{ar}}\right) ; 135.2\left(\mathrm{~d}, \mathrm{~J}=19.6 \mathrm{~Hz}, \mathrm{C}_{\mathrm{ar}, \text { quat }}\right)$; $167.7(\mathrm{~d}, \mathrm{~J}=20.8 \mathrm{~Hz}, \mathrm{C}=\mathrm{O}) . \mathbf{I R}\left(\mathrm{NaCl}, \mathrm{cm}^{-1}\right): v_{\mathrm{C}=\mathrm{O}} 1649 ; v_{\mathrm{N}-\mathrm{H}} 3309 . \mathbf{M S}(\mathrm{ES}+), \mathrm{m} / \mathrm{z}(\%): 196$ $\left(\mathrm{M}+\mathrm{H}^{+}, 100\right)$. Copies of ${ }^{1} \mathrm{H}$ NMR and ${ }^{13} \mathrm{C}$ NMR spectra are included in chapter III of the supporting information file.

$N$-Propyl-2-fluoro-2-phenylacetamide $15 \mathrm{~b} \mathrm{Mp}: 76-78{ }^{\circ} \mathrm{C}$. Flash chromatography: (hexane/EtOAc 90/10, $\left.\mathrm{R}_{\mathrm{f}}=0.14\right)$, yield 64\%. White crystals. ${ }^{1} \mathbf{H}$ NMR $\left(300 \mathrm{MHz}, \mathrm{CDCl}_{3}\right)$ : $\delta$ $0.95\left(3 \mathrm{H}, \mathrm{t}, \mathrm{J}=7.4 \mathrm{~Hz}, \mathrm{CH}_{3}\right) ; 1.49-1.66\left(2 \mathrm{H}, \mathrm{m}, \mathrm{CH}_{3} \mathrm{CH}_{2}\right) ; 3.31\left(2 \mathrm{H}, \mathrm{q}, \mathrm{J}=6.7 \mathrm{~Hz}, \mathrm{CH}_{2} \mathrm{~N}\right)$; $5.77(1 \mathrm{H}, \mathrm{d}, \mathrm{J}=48.4 \mathrm{~Hz}, \mathrm{CHF}) ; 6.53(1 \mathrm{H}, \mathrm{s}(\mathrm{br}), \mathrm{NH}) ; 7,36-7,49\left(5 \mathrm{H}, \mathrm{m}, 5 \mathrm{xCH}_{\mathrm{ar}}\right) .{ }^{19} \mathbf{F} \mathbf{~ N M R}$ $\left(282 \mathrm{MHz}, \mathrm{CDCl}_{3}\right): \delta-176.71(1 \mathrm{~F}, \mathrm{~d}, \mathrm{~J}=48.7 \mathrm{~Hz}) .{ }^{13} \mathbf{C} \mathbf{N M R}\left(300 \mathrm{MHz}, \mathrm{CDCl}_{3}\right): \delta 11.5$ $\left(\mathrm{CH}_{3}\right) ; 23,0\left(\mathrm{CH}_{3} \underline{\mathrm{CH}}_{2}\right) ; 41.1\left(\mathrm{CH}_{2} \mathrm{~N}\right) ; 52,4\left(\mathrm{~d}, \mathrm{~J}=10,4 \mathrm{~Hz}, \mathrm{NCH}_{2}\right) ; 92.2(\mathrm{~d}, \mathrm{~J}=186,9 \mathrm{~Hz}$, $\mathrm{CHF}) ; 126,9\left(\mathrm{~d}, \mathrm{~J}=6.9 \mathrm{~Hz}, 2 \mathrm{xCH}_{\mathrm{ar}}\right) ; 128,9\left(2 \mathrm{xCH}_{\mathrm{ar}}\right) ; 129,6\left(\mathrm{~d}, \mathrm{~J}=2.3 \mathrm{~Hz}, \mathrm{CH}_{\mathrm{ar}}\right) ; 135,2(\mathrm{~d}, \mathrm{~J}=$ $\left.18,5 \mathrm{~Hz}, \mathrm{C}_{\text {ar,quat }}\right) ; 168.7(\mathrm{~d}, \mathrm{~J}=21.9 \mathrm{~Hz}, \mathrm{C}=\mathrm{O}) . \mathbf{I R}\left(\mathrm{NaCl}, \mathrm{cm}^{-1}\right): v_{\mathrm{C}=\mathrm{O}} 1665 ; v_{\mathrm{N}-\mathrm{H}} 3309 . \mathbf{M S}$ $(\mathrm{ES}+), \mathrm{m} / \mathrm{z}(\%): 196\left(\mathrm{M}+\mathrm{H}^{+}, 100\right)$. Copies of ${ }^{1} \mathrm{H} \mathrm{NMR}$ and ${ }^{13} \mathrm{C}$ NMR spectra are included in chapter III of the supporting information file. 
$N$-Isopropyl-2-chloro-2-phenylacetamide 16b. The synthesis of amide $\mathbf{1 6 b}$ is given as a representative example for the synthesis of amides 16. In a $25 \mathrm{~mL}$ flask, a solution of 0.5 $\mathrm{g}$ of aziridine $12 \mathrm{c}(2.5 \mathrm{mmol})$ with $0.5 \mathrm{~mL}$ of $12 \mathrm{~N} \mathrm{HCl}$ in $10 \mathrm{~mL}$ of $\mathrm{CH}_{3} \mathrm{CN}$ was refluxed for $5 \mathrm{~h}$. Subsequently, the mixture was poured in $10 \mathrm{~mL}$ of a saturated $\mathrm{NaHCO}_{3}$ solution. After separation of the organic layer, the aqueous phase was extracted 2 times with dichloromethane. The combined organic layers were dried over $\mathrm{MgSO}_{4}$. Filtration of the drying agent and evaporation of the solvent in vacuo yielded the crude amide $\mathbf{1 6 b}$. Purification was established with column chromatography on silica gel. Mp: $107{ }^{\circ} \mathrm{C}$. Flash chromatography: (hexane/EtOAc 95/5, $\mathrm{R}_{\mathrm{f}}=0.12$ ), yield $30 \%$. White crystals. ${ }^{1} \mathbf{H}$ NMR $\left(300 \mathrm{MHz}, \mathrm{CDCl}_{3}\right): \delta 1.20\left(3 \mathrm{H}, \mathrm{d}, \mathrm{J}=6.3 \mathrm{~Hz}, \mathrm{CH}\left(\mathrm{CH}_{3}\right) \mathrm{CH}_{3}\right) ; 1.22(3 \mathrm{H}, \mathrm{d} \mathrm{J}=6.6 \mathrm{~Hz}$, $\left.\mathrm{CH}\left(\mathrm{CH}_{3}\right) \underline{\mathrm{H}}_{3}\right) ; 4.02-4.18\left(1 \mathrm{H}, \mathrm{m}, \mathrm{CH}\left(\mathrm{CH}_{3}\right)_{2}\right) ; 5.33(1 \mathrm{H}, \mathrm{s}, \mathrm{CHCl}) ; 6.61(1 \mathrm{H}, \mathrm{s}(\mathrm{br}), \mathrm{NH}) ; 7.30-$ $7.42\left(5 \mathrm{H}, \mathrm{m}, 5 \mathrm{xCH}_{\mathrm{ar}}\right) .{ }^{13} \mathbf{C} \mathbf{N M R}\left(300 \mathrm{MHz}, \mathrm{CDCl}_{3}\right.$, int. ref. $\left.=77.26 \mathrm{ppm}\right): \delta 22.5$ $\left(\mathrm{CH}\left(\underline{\mathrm{CH}}_{3}\right) \mathrm{CH}_{3}\right) ; 22,6\left(\mathrm{CH}\left(\mathrm{CH}_{3}\right) \underline{\mathrm{CH}}_{3}\right) ; 42.3\left(\underline{\mathrm{CH}}\left(\mathrm{CH}_{3}\right)_{2}\right) ; 61.8(\mathrm{CHCl}) ; 127.9\left(2 \mathrm{xCH}_{\mathrm{ar}}\right) ; 129.0$ $\left(2 \mathrm{xCH}_{\mathrm{ar}}\right) ; 129.1\left(\mathrm{CH}_{\mathrm{ar}}\right) ; 137.4\left(\mathrm{C}_{\mathrm{ar}, \text { quat }}\right) ; 166.6(\mathrm{C}=\mathrm{O})$. IR $\left(\mathrm{KBr}, \mathrm{cm}^{-1}\right): v_{\mathrm{C}=\mathrm{O}} 1659 ; v_{\mathrm{N}-\mathrm{H}} 3296$. MS (ES +$), \mathrm{m} / \mathrm{z}(\%): 212 / 14\left(\mathrm{M}+\mathrm{H}^{+}, 100 / 47\right)$. Copies of ${ }^{1} \mathrm{H}$ NMR and ${ }^{13} \mathrm{C}$ NMR spectra are included in chapter III of the supporting information file.

$N$-Isopropyl-2-chlorodecanamide 16a. The synthesis of amide 16a is analogous to the synthesis of amide $\mathbf{1 6 b}$, but the solvent used was DMSO instead of $\mathrm{CH}_{3} \mathrm{CN}$. Flash chromatography (hexane/EtOAc 95/5, $\mathrm{R}_{\mathrm{f}}=0.18$ ), yield 21\%. Mp: 61.0-61.4 ${ }^{\circ} \mathrm{C} .{ }^{1} \mathbf{H}$ NMR $\left(\mathrm{CDCl}_{3}\right): \delta 0.88\left(3 \mathrm{H}, \mathrm{t}, \mathrm{J}=6.8 \mathrm{~Hz}, \mathrm{CH}_{3}\right), 1.18\left(3 \mathrm{H}, \mathrm{d}, \mathrm{J}=6.6 \mathrm{~Hz}, \mathrm{CH}_{3}\right), 1.19(3 \mathrm{H}, \mathrm{d}, \mathrm{J}=6.6 \mathrm{~Hz}$, $\left.\mathrm{CH}_{3}\right), 1.22-1.60\left(10 \mathrm{H}, \mathrm{m}, 5 \times \mathrm{CH}_{2}\right), 1.84-1.97(1 \mathrm{H}, \mathrm{m}, \mathrm{CHaHb}), 2.02-2.18(1 \mathrm{H}, \mathrm{m}, \mathrm{CHaHb})$, 3.99-4.14 (1H, m, NCH), 4.31 (dd, J = 8.3Hz, J =4.1Hz, CHCl), 6.39 (d(br), J = 6.6Hz, NH). ${ }^{13} \mathbf{C ~ N M R}\left(\mathrm{CDCl}_{3}\right): \delta 14.2\left(\mathrm{CH}_{3}\right), 22.6$ and $22.7\left(\mathrm{CH}_{2}\right.$ and $\left.2 \times \mathrm{CH}_{3}\right), 25.8\left(\mathrm{CH}_{2}\right), 28.9\left(\mathrm{CH}_{2}\right)$, $29.2\left(\mathrm{CH}_{2}\right), 29.4\left(\mathrm{CH}_{2}\right), 31.9\left(\mathrm{CH}_{2}\right), 35.7\left(\mathrm{CH}_{2}\right), 42.0\left(\mathrm{CH}_{2}\right), 61.7(\mathrm{CHCl}), 168.2(\mathrm{C}=\mathrm{O})$. IR $(\mathrm{KBr}): v_{\max } 1470,1210 \mathrm{~cm}^{-1}$. MS (ES+), m/z(\%): $234\left(\mathrm{M}+\mathrm{H}^{+}, 100\right)$. Copies of ${ }^{1} \mathrm{H}$ NMR and ${ }^{13} \mathrm{C}$ NMR spectra are included in chapter III of the supporting information file. 